Evolutionary Selection in Normal-Form Games

Author(s): Klaus Ritzberger and Jörgen W. Weibull

Source: Econometrica, Vol. 63, No. 6 (Nov., 1995), pp. 1371-1399

Published by: The Econometric Society

Stable URL: http://www.jstor.org/stable/2171774

Accessed: 10/12/2010 18:49

Your use of the JSTOR archive indicates your acceptance of JSTOR's Terms and Conditions of Use, available at http://www.jstor.org/page/info/about/policies/terms.jsp. JSTOR's Terms and Conditions of Use provides, in part, that unless you have obtained prior permission, you may not download an entire issue of a journal or multiple copies of articles, and you may use content in the JSTOR archive only for your personal, non-commercial use.

Please contact the publisher regarding any further use of this work. Publisher contact information may be obtained at http://www.jstor.org/action/showPublisher?publisherCode=econosoc.

Each copy of any part of a JSTOR transmission must contain the same copyright notice that appears on the screen or printed page of such transmission.

JSTOR is a not-for-profit service that helps scholars, researchers, and students discover, use, and build upon a wide range of content in a trusted digital archive. We use information technology and tools to increase productivity and facilitate new forms of scholarship. For more information about JSTOR, please contact support@jstor.org. 


\title{
EVOLUTIONARY SELECTION IN NORMAL-FORM GAMES
}

\author{
By Klaus Ritzberger and JöRgen W. Weibull ${ }^{1}$
}

\begin{abstract}
This paper investigates stability properties of evolutionary selection dynamics in normal-form games. The analysis is focused on deterministic dynamics in continuous time and on asymptotic stability of sets of population states, more precisely of faces of the mixed-strategy space. The main result is a characterization of those faces which are asymptotically stable in all dynamics from a certain class, and we show that every such face contains an essential component of the set of Nash equilibria, and hence a strategically stable set in the sense of Kohlberg and Mertens (1986).
\end{abstract}

KEYwORDS: Dynamics, evolution, noncooperative games, stability.

\section{INTRODUCTION}

MOST APPLICATIONS OF NONCOOPERATIVE GAME THEORY build on such solution concepts as Nash equilibrium. As is well known by now, the rationalistic foundation of this approach is quite demanding. Not only is it required that agents be optimizers, but it also presumes a large degree of coordination of different agents' expectations (see, e.g., Tan and Werlang (1988), and Aumann and Brandenburger (1992)). In recent years researchers have investigated alternative foundations of Nash equilibrium play. Particularly promising seems the approach taken in evolutionary game theory. Instead of asking if agents are rational in some epistemologically well-defined sense, one asks if evolutionary selection processes induce a tendency towards aggregate Nash equilibrium behavior. In other words, one then investigates the validity of Friedman's (1953) "as if" paradigm in the context of strategic interaction.

The idea of an "as if" interpretation of equilibrium points, however, dates back to the early days of Nash equilibrium:

\footnotetext{
"We shall now take up the "mass-action" interpretation of equilibrium points. ... It is unnecessary to assume that the participants have full knowledge of the total structure of the game, or the ability and inclination to go through any complex reasoning processes. But the participants are supposed to accumulate empirical information on the relative advantages of the various pure strategies at their disposal.

To be more detailed, we assume that there is a population (in the sense of statistics) of participants for each position of the game. Let us also assume that the "average playing" of the game involves $n$ participants selected at random from the $n$ populations, and that there is a stable average frequency with which each pure strategy is employed by the "average member" of the appropriate population.

Since there is to be no collaboration between individuals playing in different positions of the game, the probability that a particular $n$-tuple of pure strategies will be employed in a playing of the game should be the product of the probabilities indicating the chance of each of the $n$ pure strategies to be employed in a random playing. ...
}

${ }^{1}$ Both authors gratefully acknowledge helpful comments from Jonas Björnerstedt, Josef Hofbauer, a co-editor, and three anonymous referees, and thank the Industrial Institute for Economic and Social Research, Stockholm, and the Institute for Advanced Studies, Vienna, for their hospitality and sponsorship of this research. 
Thus the assumptions we made in this "mass-action" interpretation lead to the conclusion that the mixed strategies representing the average behavior in each of the populations form an equilibrium point. ...

Actually, of course, we can only expect some sort of approximate equilibrium, since the information, its utilization, and the stability of the average frequencies will be imperfect." (Nash (1950, p. 21-23).)

There are several approaches to formalize the above ideas. One is to investigate the dynamics of social evolution. The best studied setting for evolutionary dynamics was originally motivated by biology and concerns pairwise random matchings of individuals drawn from a single infinitely large population and playing a symmetric two-player game. In the so-called replicator dynamics (Taylor and Jonker (1978)) individuals change from currently worse to better strategies at rates which are proportional to current payoff differences. Results for the single-population case are encouraging for the Nash equilibrium paradigm. Weak dynamic stability in the replicator dynamics was shown to imply symmetric Nash equilibrium behavior (Bomze (1986)) and, for a broad class of dynamics, it was established that convergence to a stationary state from an interior initial state also implies Nash equilibrium behavior (Nachbar (1990)).

However, many economic applications call for multi-population, rather than single-population dynamics. For instance, the player roles may be those of "buyers" and "sellers," each type of individual being drawn from his or her "player-role population." Moreover, in most applications, the game will not be symmetric and may involve more than two players. Thus one is lead to study evolutionary selection dynamics in $n$-player games, in which each player role is represented by a distinct population in the spirit of Nash's "mass-action" interpretation - the topic of the present paper.

Individuals are randomly drawn from infinitely large populations also in the multi-population setting. Each matching involves one individual from each of the player-populations and each individual is "programmed" to use a pure strategy available to the player whose role she plays. Over time the strategy distribution in each player population changes according to some dynamic selection process. The present paper studies classes of such dynamics all of which include the replicator dynamics, as well as a family of imitation dynamics. And indeed, the earlier positive results from the symmetric single-population setting do carry over to the (symmetric and asymmetric) multi-population case. Again weak dynamic stability properties and convergence imply Nash equilibrium behavior (Friedman (1991), Samuelson and Zhang (1992); see Section 3 below for details).

There is an important caveat to these positive results, viz. that few Nash equilibria have strong stability properties in multi-population dynamics. More precisely, only strict equilibria are asymptotically stable in the replicator dynamics (Ritzberger and Vogelsberger (1990, Proposition 1)), and virtually only strict equilibria are asymptotically stable in so-called aggregate monotonic selection dynamics (Samuelson and Zhang (1992, Theorem 4 and Corollary 1)). Consequently, many games possess no asymptotically stable equilibrium at all. Hence, 
the connection between evolutionary selection in $n$-player games and Nash equilibrium is weaker than it may first appear.

Since these negative results concern individual points in the strategy space, it is natural to turn to sets instead. Nontrivial connected sets of Nash equilibria are endemic in normal-form games that derive from extensive forms, and part of the refinement literature has also turned to set-valued solution concepts in order to cope with existence problems.

There are a number of set-valued approaches in the literature on evolutionary game theory as well. Thomas (1985) develops a set-valued generalization of the static concept of evolutionarily stable strategies (Maynard Smith and Price (1973)). Swinkels (1992a, 1992b) also proposes set-valued generalizations of evolutionarily stable strategies and shows that those sets contain sets of Nash equilibria which meet certain refinement criteria. Swinkels (1993) deduces the latter from dynamic asymptotic stability of sets in a selection dynamics from a broad class.

In the present paper we focus on asymptotic stability of a certain class of strategy sets, namely faces of the mixed strategy space of the game. A face is the Cartesian product of sets of mixed strategies, one set for each player, each of which consists of all mixtures over some subset of the player's pure strategy set. At one extreme end of this spectrum there are individual pure strategy combinations (minimal faces). The opposite extreme is the set of all mixed strategy combinations in the game (the maximal face). Dynamic stability properties of faces can thus be associated with predictions in terms of subsets of pure strategies, one subset for each player position.

The focus on asymptotic stability is due to the fact that this property, unlike the weaker criterion of Lyapunov stability, is a "structurally robust" property in the sense that it is not destroyed by small perturbations of (the vector field of) the dynamics. In particular, asymptotic stability is preserved even if small fractions of a population occasionally "mutate," "experiment," or "make mistakes," thus generating small shocks to the population state.

A similar robustness criterion motivates the focus on faces. Since in many applications only broad qualitative features of the selection dynamics are know or assumed, it is desirable that the sets under consideration be dynamically stable in a fairly wide class of selection dynamics. We show that such broader robustness of dynamic stability properties disqualifies all (closed) sets in the (relative) interior of any face. Hence, when such robustness is called for, the restriction to sets which are faces is not so severe, and asymptotic stability provides predictions which are immune to small amounts of (unmodeled) drift.

Our main result is a characterization of all faces which are set-wise asymptotically stable in all dynamics from a class of dynamics which contains all aggregate monotonic selection dynamics. The characterizing criterion is that the set in question be "closed" under a certain correspondence which we call the "betterreply" correspondence - in analogy with the well-known best-reply correspondence used in noncooperative game theory. This "new" correspondence assigns to each mixed strategy combination $\sigma$ those pure strategies for each player which give that player at least the same payoff as she has in $\sigma$. Such pure 
strategies are thus weakly better replies to $\sigma$ than $\sigma$ is itself. We call a product set of pure strategies closed under the better-reply correspondence if the image under this correspondence of every mixed strategy combination with support in the set is contained in the set. (Such a set is necessarily "closed under rational behavior," or a curb set; see Basu and Weibull (1991).)

The equivalence of closure under the better-reply correspondence and asymptotic stability of the associated face provides an operational criterion by means of which pure strategy subsets spanning an asymptotically stable face can be identified, a criterion based exclusively on the data of the game. In particular examples this criterion has significant cutting power (see Section 6). However, as yet the strength of the criterion in general remains an open issue. Therefore, there may be many games where few pure strategies can be "robustly" rejected on grounds of dynamic social evolution as modeled here.

There is a stark contrast between "robust" evolutionary predictions and noncooperative game theory concerning completely mixed Nash equilibria. For while these pass all the Nash equilibrium refinements based on strategy perturbations ("trembles"), no such equilibrium is robustly stable in the present class of evolutionary selection dynamics. More precisely, even if such an equilibrium is dynamically stable in some aggregate monotonic selection dynamics, it is unstable in others. Hence, unless we are convinced that "real life" adaption is well described by dynamics in the "stable subclass," we may have to live with the possibility of ever-lasting oscillations in certain aggregate social behaviors. ${ }^{2}$

We also show that a face spanned by a product set of pure strategies which is closed under the better-reply correspondence contains an essential component of Nash equilibria. That is, it contains a closed and connected set of Nash equilibria such that every nearby game, in terms of pure strategy payoffs, has a nearby Nash equilibrium. This is one of the most stringent set-wise refinements from the noncooperative game theory literature, implying strategic stability in the sense of Kohlberg and Mertens (1986).

Put together with the above characterization result, one obtains that an asymptotically stable face contains an essential component of Nash equilibria. This implication parallels Theorem 1 in Swinkels (1993). He shows that if a set is asymptotically stable in a selection dynamics from a broad class, and meets a certain topological condition, met by any face, then it contains a hyperstable set of Nash equilibria in the sense of Kohlberg and Mertens (1986).

The material is organized as follows. Section 2 contains notation and basic definitions. Section 3 provides, in a unified and sometimes more general form, essentially known results on point-wise stability (except for Propositions 2 and 3). All proofs for this section have been relegated to an Appendix at the end of the paper. In Section 4 we elaborate on a class of correspondences which we call behavior correspondences, of which the best-reply and better-reply correspon-

\footnotetext{
${ }^{2}$ Even if a solution persistently oscillates the time average of the population state may be of significance. In games with a unique interior Nash equilibrium, for instance, it converges under the replicator dynamics to the equilibrium, provided the closure of the orbit is interior (cf. Hofbauer and Sigmund (1988)).
} 
dences are instances, and we relate sets closed under such correspondences to the notions of strict and non-strict Nash equilibrium. Our main result is given in Section 5. Section 6 contains examples of the concepts used. Some directions for further research are mentioned in Section 7.

\section{NOTATION AND DEFINITIONS}

\subsection{The Game}

Let $\Gamma$ be a normal-form game with player set $\mathscr{N}=\{1,2, \ldots, n\}$, for some positive integer $n$, and with $S=\times_{i \in \mathscr{N}} S_{i}$ as the set of pure strategy combinations $s=\left(s_{1}, s_{2}, \ldots, s_{n}\right)$, where each set $S_{i}$ consists of $K_{i}$ pure strategies $s_{i}^{k}$, $k=1,2, \ldots, K_{i}$, available to player $i \in \mathscr{N}$. The set of mixed strategies of player $i$

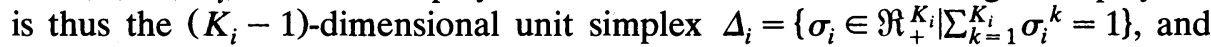
$\Delta=\times_{i \in \mathscr{N}} \Delta_{i}$ is the polyhedron of mixed strategy combinations $\sigma=\left(\sigma_{1}, \ldots, \sigma_{n}\right)$ in the game. We identify each pure strategy $s_{i}^{k} \in S_{i}$ with the corresponding unit vector $e_{i}^{k} \in \Delta_{i}$ (hence $S_{i}$ is the subset of vertices of $\Delta_{i}$ ). The support of some mixed strategy $\sigma_{i} \in \Delta_{i}$ is denoted by $\operatorname{supp}\left(\sigma_{i}\right)=\left\{s_{i}^{k} \in S_{i} \mid \sigma_{i}^{k}>0\right\}$. The mapping $u: S \rightarrow \Re^{n}$ gives the payoffs of pure strategy combinations, and the multilinear expected payoff function $U: \Delta \rightarrow \Re^{n}$ is defined in the usual manner.

Let $\beta=X_{i \in \mathscr{N}} \beta_{i}: \Delta \rightarrow S$ be the pure best-reply correspondence which maps mixed strategy combinations to their pure best-reply strategy combinations. More precisely, for each player $i \in \mathscr{N}$ and strategy combination $\sigma \in \Delta$,

$$
\beta_{i}(\sigma)=\left\{s_{i}^{k} \in S_{i} \mid U_{i}\left(\sigma_{-i}, s_{i}^{k}\right) \geq U_{i}\left(\sigma_{-i}, s_{i}\right), \forall s_{i} \in S_{i}\right\} .
$$

The correspondence assigning mixed best replies is denoted $\tilde{\beta}=\times_{i \in \mathscr{N}} \tilde{\beta}_{i}$, where

$$
\tilde{\beta}_{i}(\sigma)=\left\{\tilde{\sigma}_{i} \in \Delta_{i} \mid \operatorname{supp}\left(\tilde{\sigma}_{i}\right) \subset \beta_{i}(\sigma)\right\} .
$$

It is well known that both $\beta$ and $\tilde{\beta}$ are u.h.c. correspondences on $\Delta$.

A Nash equilibrium is a strategy combination $\sigma \in \Delta$ which is a fixed point of $\tilde{\beta}$. The set of Nash equilibria of a game $\Gamma$ is denoted by

$$
E(\Gamma)=\{\sigma \in \Delta \mid \sigma \in \tilde{\beta}(\sigma)\} .
$$

A strict equilibrium is a strategy combination $\sigma \in \Delta$ which is its own unique best 'reply, i.e., such that $\{\sigma\}=\tilde{\beta}(\sigma)$. Every strict equilibrium $\sigma \in E(\Gamma)$ is pure, so one may view it as a fixed point of $\beta$ in $S$.

\subsection{Dynamics}

A regular selection dynamics on $\Delta$ is a system of ordinary differential equations (time indices suppressed)

$$
\dot{\sigma}_{i}^{k}=f_{i}^{k}(\sigma) \sigma_{i}^{k}, \quad \forall k=1, \ldots, K_{i}, \forall i \in \mathscr{N},
$$


with $f_{i}: \Delta \rightarrow \Re^{K_{i}}, \forall i \in \mathscr{N}$, and $f=\times_{i \in \mathscr{N}} f_{i}$ is such that

(i) $f$ is Lipschitz continuous on $\Delta$,

(ii) $f_{i}(\sigma) \cdot \sigma_{i}=0, \forall \sigma \in \Delta, \forall i \in \mathscr{N}^{3}$

As a consequence of the Picard-Lindelöf Theorem the above system has a unique solution $\sigma\left(., \sigma^{o}\right): \Re \rightarrow \Delta$ through every initial state $\sigma^{o} \in \Delta, \sigma\left(t, \sigma^{o}\right) \in$ $\Delta$ denoting the state at time $t \in \Re$. All regular selection dynamics have the property that extinct strategies stay extinct forever.

The best studied regular selection dynamics in evolutionary game theory is the so-called replicator dynamics. For an $n$-player game $\Gamma$, this dynamics is usually defined by the following system of (quadratic) ordinary differential equations on the polyhedron $\Delta$ :

$$
\dot{\sigma}_{i}^{k}=\left[U_{i}\left(\sigma_{-i}, s_{i}^{k}\right)-U_{i}(\sigma)\right] \sigma_{i}^{k}, \quad \forall k=1, \ldots, K_{i}, \forall i \in \mathscr{N}
$$

(see, e.g., Taylor (1979), Zeeman (1980), Hofbauer and Sigmund (1988), Friedman (1991), Samuelson and Zhang (1992)). Alternatively the replicator dynamics is sometimes written

$$
\dot{\sigma}_{i}^{k}=\frac{1}{U_{i}(\sigma)}\left[U_{i}\left(\sigma_{-i}, s_{i}^{k}\right)-U_{i}(\dot{\sigma})\right] \sigma_{i}^{k}, \quad \forall k=1, \ldots, K_{i}, \forall i \in \mathscr{N},
$$

where the average payoff to each population $i, U_{i}(\sigma)$, is taken to be positive (Maynard Smith (1982, p. 200), Hofbauer and Sigmund (1988, p. 273)). ${ }^{4}$ In the biology literature, "there is ... room for doubt as to which form is more appropriaie" (Maynard Smith (1982, p. 201)). Following the economics literature (Samuelson and Zhang (1992, p. 370), Swinkels (1993, p. 466)) we will hereafter refer to the first and more common version as the replicator dynamics. The second version will be called the payoff-normalized replicator dynamics. As will be seen below, many of our results hold for classes of evolutionary selection dynamics which contain both versions.

An aggregate monotonic selection (AMS) dynamics (Samuelson and Zhang (1992)) is a regular selection dynamics such that for all $i \in \mathscr{N}, \sigma \in \Delta$, and $\sigma_{i}^{\prime}, \sigma_{i}^{\prime \prime} \in \Delta_{i}$,

$$
U_{i}\left(\sigma_{-i}, \sigma_{i}^{\prime}\right)<U_{i}\left(\sigma_{-i}, \sigma_{i}^{\prime \prime}\right) \Leftrightarrow f_{i}(\sigma) \cdot \sigma_{i}^{\prime}<f_{i}(\sigma) \cdot \sigma_{i}^{\prime \prime} .
$$

By a straightforward generalization of Theorem 3 in Samuelson and Zhang (1992, pp. 374) it can be shown that any AMS dynamics can be written in the form

$$
f_{i}^{k}(\sigma)=\omega_{i}(\sigma)\left[U_{i}\left(\sigma_{-i}, s_{i}^{k}\right)-U_{i}(\sigma)\right], \quad \forall k=1, \ldots, K_{i}, \forall i \in \mathscr{N}
$$

\footnotetext{
${ }^{3}$ This definition can be shown to be equivalent to the one given by Samuelson and Zhang (1992, p. 368$)$.

${ }^{4}$ We are grateful to an anonymous referee for pointing this out.
} 
for some positive function $\omega_{i}: \Delta \rightarrow \Re_{++}$, for every $i \in \mathscr{N}$. The class of AMS dynamics is thus given by all "player-specific reparameterizations of time" in the replicator dynamics, the latter being the special case $\omega_{i}(\sigma)=1, \forall \sigma \in \Delta, \forall i \in \mathscr{N}$. The payoff-normalized replicator dynamics is the special case with $\omega_{i}(\sigma)=$ $1 / U_{i}(\sigma)>0, \forall \sigma \in \Delta, \forall i \in \mathscr{N}$.

A weaker condition than aggregate monotonicity is to require the equivalence to hold only for pure strategies $\sigma_{i}^{\prime}=s_{i}^{\prime} \in S_{i}$ and $\sigma_{i}^{\prime \prime}=s_{i}^{\prime \prime} \in S_{i}$. Such a selection dynamics is called monotonic:

$$
U_{i}\left(\sigma_{-i}, s_{i}^{k}\right)<U_{i}\left(\sigma_{-i}, s_{i}^{h}\right) \Leftrightarrow f_{i}^{k}(\sigma)<f_{i}^{h}(\sigma),
$$

for all $i \in \mathscr{N}, \sigma \in \Delta$, and $s_{i}^{k}, s_{i}^{h} \in S_{i}$.

Another class of selection dynamics which also contains all AMS dynamics is the following: A sign-preserving selection (SPS) dynamics is a regular selection dynamics such that for all $i \in \mathscr{N}$, all $\sigma \in \Delta$, and all $s_{i}^{k} \in \operatorname{supp}\left(\sigma_{i}\right)$

$$
U_{i}\left(\sigma_{-i}, s_{i}^{k}\right)<U_{i}(\sigma) \Leftrightarrow f_{i}^{k}(\sigma)<0 .
$$

In other words: Strategies which are at least as good as the average grow at nonnegative rates, and strategies which perform worse than average are precisely those which are selected against. ${ }^{5}$ This class of selection dynamics contains all AMS dynamics and turns out to be of special significance for the present approach.

For a SPS dynamics the ordering of growth rates of population shares is not determined from payoffs, while for a monotonic selection dynamics it is. On the other hand, for a monotonic selection dynamics the signs of growth rates of population shares of strategies that do neither best nor worst are not determined, while for a SPS dynamics they are. So, though the intersection of the class of SPS dynamics and monotonic selection dynamics is nonempty, the two are distinct classes.

Aggregate monotonicity, which is thus a special case of both monotonic and sign-preserving selection dynamics, can be derived from underlying microeconomic models that assume a certain imitative adaptation of the behavior of individuals. In large populations one may imagine the following scenario (for details see Björnerstedt and Weibull (1993)): While at each instant of time every individual is bound to use a particular pure strategy, occasionally one or the other individual reviews her or his strategy choice. Suppose these "arrival times" are governed by independent Poisson processes, which may depend on the current population state. Once an individual reviews her strategy, she will switch to a new pure strategy with a conditional probability that may depend positively on its current success (payoff) and popularity (population frequency). Note that such an adaption process does not presume any knowledge about payoffs to other player positions in the game. If, in particular, individuals with currently less successful strategies review their strategy choice at a linearly higher rate

\footnotetext{
${ }^{5}$ A similar, though slightly stronger definition of "sign-preserving" dynamics appears in Nachbar (1990).
} 
than individuals with more successful strategies, and/or the conditional switching probability towards any pure strategy is proportional to its popularity, with the proportionality factor linearly increasing in the strategy's current success, then an AMS dynamics results. In particular, both the replicator dynamics and its payoff-normalized version can be so derived. Without the linearity assumptions these imitation processes merely result in SPS or monotonic selection dynamics, depending on the nature of the imitation mechanism.

Given some regular selection dynamics on the polyhedron $\Delta$ of mixed strategy combinations, a set $A \subset \Delta$ is called positively invariant if any solution path starting in $A$ remains in $A$ for the entire future: $\sigma\left(t, \sigma^{o}\right) \in A, \forall \sigma^{o} \in A$, $\forall t \in \Re_{+}$. It is called invariant if, moreover, any solution path in $A$ has also been in $A$ for the entire past, $\sigma\left(t, \sigma^{o}\right) \in A, \forall \sigma^{o} \in A, \forall t \in \Re$. A point $\sigma^{*} \in \Delta$ is called stationary or a rest point, if $\left\{\sigma^{*}\right\} \subset \Delta$ is an invariant set. Both $\Delta$ and its interior $\operatorname{int}(\Delta)$ are invariant in every regular selection dynamics. ${ }^{6}$

Let $F(\Gamma) \subset \Delta$ be the set of rest points in a given regular selection dynamics. It is easily verified that the set $F(\Gamma)$ of all rest points is the same for all SPS and all monotonic selection dynamics and is given by

$$
F(\Gamma)=\left\{\sigma \in \Delta \mid U_{i}\left(\sigma_{-i}, s_{i}\right)=U_{i}(\sigma), \forall s_{i} \in \operatorname{supp}\left(\sigma_{i}\right), \forall i \in \mathscr{N}\right\}
$$

A closed invariant set $A \subset \Delta$ is said to be (Lyapunov) stable, if the solution curves remain arbitrarily close to $A$ for all initial states sufficiently close to $A$. Formally, a neighborhood $\mathscr{B}$ of a closed set $A \subset \Delta$ is an open set containing $A$, and:

Definition: A closed invariant set $A \subset \Delta$ is (Lyapunov) stable, if for every neighborhood $\mathscr{B}^{\prime}$ of $A$ there exists a neighborhood $\mathscr{B}^{\prime \prime}$ of $A$ such that $\sigma\left(t, \sigma^{o}\right) \in \mathscr{B}^{\prime}$ for all $\sigma^{o} \in \mathscr{B}^{\prime \prime} \cap \Delta$ and all $t \geq 0$.

A more stringent stability notion is that of asymptotic stability. It requires on top of (Lyapunov) stability that the set $A$ be a local attractor in the sense that all dynamic paths starting sufficiently close to $A$ converge to $A$ over time. Formally, we have the following definition:

Definition: A closed invariant set $A \subset \Delta$ is asymptotically stable if it is stable and there exists some neighborhood $\mathscr{B}$ of $A$ such that $\sigma\left(t, \sigma^{o}\right) \rightarrow_{t \rightarrow \infty} A$, for all $\sigma^{o} \in \mathscr{B} \cap \Delta$. $^{7}$

Since any stationary point $\sigma \in \Delta$ constitutes a closed subset $\{\sigma\}$ of $\Delta$, the above definitions also cover stability notions for points. The induced stability criteria for points coincide with the standard definitions (see, e.g., Hirsch and Smale (1974, pp. 185)).

\footnotetext{
${ }^{6}$ Since $\Delta$ is lower-dimensional than the embedding Euclidean space, it has empty interior. But $\Delta$ has a nonempty relative interior, denoted $\operatorname{int}(\Delta)$. A subset of $\Delta$ is called relatively interior if it is a subset of $\operatorname{int}(\Delta)$.

${ }^{7}$ The notation $\sigma\left(t, \sigma^{o}\right) \rightarrow_{t \rightarrow \infty} A$ is used to mean $\min _{a \in A}\left\|\sigma\left(t, \sigma^{o}\right)-a\right\| \rightarrow_{t \rightarrow \infty} 0$, where $\|\cdot\|$ denotes the Euclidean metric.
} 
For a given game $\Gamma$, two player positions $i, j \in \mathscr{N}$ are said to be symmetric, if $S_{i}=S_{j}$ and $U_{i}\left(s_{-i j}, s_{i}^{k}, s_{j}^{h}\right)=U_{j}\left(s_{-i j}, s_{i}^{h}, s_{j}^{k}\right), \forall k, h=1, \ldots, K_{i}=K_{j}, \forall s_{-i j} \in S_{-i j}$. If $i$ and $j$ are symmetric player positions, then $U_{i}\left(\sigma_{-i}, s_{i}^{k}\right)=U_{j}\left(\sigma_{-j}, s_{j}^{k}\right), \forall k=$ $1, \ldots, K_{i}$, and $U_{i}(\sigma)=U_{j}(\sigma)$, for all $\sigma \in \Delta$ with $\sigma_{i}=\sigma_{j}$. Hence, for any AMS dynamics which satisfies $\omega_{i}(\sigma)=\omega_{j}(\sigma), \forall \sigma \in \Delta$, symmetry of $i$ and $j$ implies that the diagonal $D_{i j}=\left\{\sigma \in \Delta \mid \sigma_{i}=\sigma_{j}\right\}$ is an invariant set (because $\sigma_{i}=\sigma_{\dot{j}} \Rightarrow \dot{\sigma}_{i}$ $=\dot{\sigma}_{j}$ ). If $\mathscr{N}=\{1,2\}$ and the two player positions are symmetric, then $\omega_{1}(\sigma) \equiv$ $\omega_{2}(\sigma)$ is simply a reparametrization of time which does not change the solution curves. So any such AMS dynamics induces the same orbits as the replicator dynamics on $D_{12} \subset \Delta_{1} \times \Delta_{2}$. This is, for instance, the case for the payoff-normalized version of the replicator dynamics. Since $D_{12}$ is isomorphic with the state space of single-population dynamics in symmetric two-player games, every AMS dynamics with $\omega_{1}=\omega_{2}$ induces the replicator dynamics in the corresponding single-population dynamics. ${ }^{8}$

\section{POINT-WISE STABILITY}

From the discussion it is clear that a Nash equilibrium is a rest point for any sign-preserving or monotonic selection dynamics (but not vice versa): $E(\Gamma) \subset$ $F(\Gamma)$. But the connection between evolutionary dynamics and Nash equilibrium extends further. For monotonic single-population selection dynamics in symmetric two-player games it has been shown that any (Lyapunov) stable state constitutes a (symmetric) Nash equilibrium, and that convergence from an initial state in which all pure strategies are present implies that the limit state corresponds to a (symmetric) Nash equilibrium (Nachbar (1990)). On the other hand, if a state is asymptotically stable in the replicator dynamics, then it corresponds to an isolated and (symmetric trembling-hand) perfect Nash equilibrium (Bomze (1986)).

These positive results carry over to multi-population dynamics in symmetric and asymmetric $n$-player games (Nachbar (1990), Friedman (1991), Samuelson and Zhang (1992)). Formally: Call a state $\sigma \in \Delta$ reachable in a selection dynamics if there exists $\sigma^{o} \in \operatorname{int}(\Delta)$ such that $\sigma\left(t, \sigma^{o}\right) \rightarrow_{t \rightarrow \infty} \sigma$. It follows from continuity that such a state $\sigma$ is stationary in the selection dynamics under consideration.

PROPOSITION 1: For any monotonic selection dynamics:

(a) If $\sigma \in \Delta$ is Lyapunov stable, then $\sigma \in E(\Gamma)$.

(b) If $\sigma \in \Delta$ is reachable, then $\sigma \in E(\Gamma)$.

Proof: See Appendix.

For predictive purposes, however, these dynamic properties appear too weak.

\footnotetext{
${ }^{8}$ By single-population dynamics we mean that interacting individuals are drawn from the same population.
} 
For if a population state is reachable but not Lyapunov stable, then arbitrary small perturbations of the state-by (unmodeled) "mutations," "mistakes," or "experiments," say-can lead the population far away. Likewise, if a population state is Lyapunov but not asymptotically stable, then certain small shocks to the state will dislocate the population state permanently (for a discussion of the related notion of "evolutionary drift" see Binmore and Samuelson (1994)). By contrast, the more stringent criterion of asymptotic stability does guarantee a "local pull" towards the state (or set) in question. In particular, asymptotic stability (of a point or set) is preserved (for some nearby point or set) even if the vector field is slightly bent inwards at the boundary of $\Delta$ as, for instance, by small amounts of mutations.

However, it has been shown that in the replicator dynamics no interior population state is asymptotically stable (cf. Amann and Hofbauer (1985); Hofbauer and Sigmund (1988, p. 282), Ritzberger and Vogelsberger (1990, Lemma 5)). Moreover, each face of $\Delta$ can be thought of as a smaller game of its own, derived from $\Gamma$ by deleting all unused pure strategies. The replicator dynamics of such a reduced game is just the replicator dynamics of $\Gamma$ restricted to the corresponding face of $\Delta$, each face being invariant. Consequently, no mixed strategy combination, in which at least one player randomizes, is asymptotically stable in the replicator dynamics.

This evolutionary instability of mixed equilibria parallels the well known "epistemic" instability of mixed equilibria in the noncooperative approach (Harsanyi (1973), van Damrie, (1987, p. 19)): In a mixed equilibrium some player can choose another randomization than the one prescribed by the equilibrium, without losses of expected payoff, given that the other players stick to their equilibrium strategies. ${ }^{9}$ If other players anticipate this possibility, then they may want to change their strategies, etc.

The dynamic instability of interior states can be shown to lead to the following characterization: ${ }^{10}$

Proposition 2: A Nash equilibrium is asymptotically stable in the replicator dynamics if and only if it is a strict equilibrium.

Proof: See Appendix.

It is well known that the single-population replicator dynamics for symmetric two-player games can have asymptotically stable rest points which are interior. This can happen if, for instance, the diagonal $D_{12}$ of $\Delta$ is contained in the

\footnotetext{
${ }^{9}$ Harsanyi, however, shows that mixed equilibria which satisfy a regularity condition (cf. Harsanyi (1973, Lemma 9)) still have a justification as limiting equilibria of games with randomly perturbed payoffs as the perturbations approach zero.

${ }^{10}$ Proposition 2 appears as Proposition 1 in Ritzberger and Vogelsberger (1990), and is a slight sharpening of Theorem 4 and Corollary 1 in Samuelson and Zhang (1992), for the case of the replicator dynamics.
} 
convergent manifold of an equilibrium which is a saddle point in the full state space $\Delta$.

Proposition 2 says that the Nash equilibria which are asymptotically stable in the multi-population replicator dynamics are precisely those which constitute the only best-reply to a neighborhood of themselves. So what are the dynamic stability properties of equilibria which are a best-reply to a neighborhood of themselves, but not the only one? Such a notion is known as a robust equilibrium (Okada (1983)). A Nash equilibrium $\sigma$ is called robust, if there exists some neighborhood $\mathscr{O}$ of $\sigma$ such that $\sigma \in \tilde{\beta}\left(\sigma^{o}\right), \forall \sigma^{o} \in \mathscr{O} \cap \Delta$. One might, therefore, expect that for robust equilibria the weaker (Lyapunov) stability criterion is satisfied. This turns out to be true within the class of sign-preserving selection (SPS) dynamics.

Proposition 3: (a) Every robust equilibrium is Lyapunov stable in any SPS dynamics.

(b) For every robust equilibrium there exists a neighborhood $\mathscr{O}$ such that $\sigma\left(t, \sigma^{o}\right) \rightarrow_{t \rightarrow \infty} E(\dot{\Gamma}) \cap \mathscr{O}, \forall \sigma^{o} \in \mathscr{O} \cap \Delta$, in any AMS dynamics.

Proof: See Appendix.

\section{BEHAVIOR CORRESPONDENCES}

Let $\Phi$ be the class of u.h.c. correspondences ${ }^{-} \varphi=\times_{i \in \mathscr{N}} \varphi_{i}: \Delta \rightarrow S$ such that $\beta(\sigma) \subset \varphi(\sigma)$ for all $\sigma \in \Delta$ (weak inclusion). Correspondences $\varphi \in \Phi$ will henceforth be called behavior correspondences. For any correspondence $\varphi: \Delta \rightarrow S$, and any nonempty set $A \subset \Delta, \varphi(A) \subset S$ denotes the (nonempty) union of all images $\varphi(\sigma)$ with $\sigma \in A$, i.e., $\varphi(A)=\bigcup_{\sigma \in A} \varphi(\sigma)$.

Let $P$ be the set of all nonempty product sets $X \subset S$, i.e., $X=\times_{i \in \mathscr{N}} X_{i}$, where $\varnothing \neq X_{i} \subset S_{i}, \forall i \in \mathscr{N}$. For any nonempty set $X_{i} \subset S_{i}$, let $\Delta_{i}\left(X_{i}\right)$ be the set of all mixed strategies with support in $X_{i}$. For any $X \in P$, let $\Delta(X)=\times_{i \in \mathscr{N}} \Delta_{i}\left(X_{i}\right)$. This is the face of the polyhedron $\Delta$ spanned by $X$. Such a face $\Delta(X)$ is itself a polyhedron of mixed strategy combinations associated with the reduced game in which the pure strategy set of player $i \in \mathscr{N}$ is $X_{i}$.

Basu and Weibull (1991) call a set $X \in P$ closed under rational behavior (curb) if it contains all its best replies, i.e., if $\beta(\Delta(X)) \subset X$, and call it tight curb if $\beta(\Delta(X))=X$. More generally: given any behavior correspondence $\varphi \in \Phi$, we here call a set $X \in P$ closed under $\varphi$ if $\varphi(\Delta(X)) \subset X$ and fixed under $\varphi$ if $\varphi(\Delta(X))=X .^{11}$ Clearly $X \in P$ is a curb set if it is closed under some behavior correspondence $\varphi \in \Phi$, by $\beta(\Delta(X)) \subset \varphi(\Delta(X)) \subset X$. A set $X \in P$ is a minimal closed set under $\varphi$ if it is closed under $\varphi$ and contains no proper subset which is closed under $\varphi$.

\footnotetext{
${ }^{11}$ The terminology is motivated by the fact that a fixed set for a correspondence is the direct generalization of a fixed point of a function, when the correspondence is viewed as a function from the power set into itself (cf. Berge (1963, p. 113)).
} 
The following lemma generalizes some basic properties of curb sets to sets which are closed under some behavior correspondence. The proof follows Basu and Weibull (1991).

LEMMA 1: (a) If $X \in P$ is a minimal closed set under $\varphi \in \Phi$, then it is a fixed set under $\varphi$.

(b) For every $\varphi \in \Phi$ there exists a minimal closed set.

(c) If a singleton set $X=\{s\}$ is closed under some $\varphi \in \Phi$, then $s \in S$ is a strict equilibrium.

Proof: (a) Suppose $X \in P$ is a minimal closed set under $\varphi \in \Phi$, but $X \neq$ $\varphi(\Delta(X))$. Then there exists some player $i \in \mathscr{N}$ such that $\varphi_{i}(\Delta(X)) \subset{ }_{\neq} X_{i}$. Let $Z_{i}=\varphi_{i}(\Delta(X))$, and $\forall j \neq i$, let $Z_{j}=X_{j}$. Clearly $\varphi(\Delta(Z)) \subset \varphi(\Delta(X)) \subset Z$, so $X$ is not minimal一a contradiction.

(b) By $\varphi(\Delta) \subset S$ the nonempty collection $Q \subset P$ of sets $X \in P$ which are closed under some given $\varphi \in \Phi$ is finite and partially ordered by set inclusion, and hence contains at least one minimal such set.

(c) If a singleton set $X=\{s\}$ is closed under $\varphi \in \Phi$, then $\varnothing \neq \beta(s) \subset \varphi(s) \subset$ $\{s\}$, and so $\beta(s)=\{s\}$, i.e., $s \in S$ is a strict equilibrium.

The next result is a key observation for the subsequent analysis. Essentially it provides a generalization of a property of strict equilibria which non-strict Nash equilibria lack, and which, in a sense, is the converse of the defining property of Nash equilibrium. While a strategy combination $\sigma \in \Delta$ is defined as a Nash equilibrium whenever it is contained in its set of best replies, $\{\sigma\} \subset \tilde{\beta}(\sigma)$, only strict equilibria have the complementary (curb) property of containing all their best replies, $\tilde{\beta}(\sigma) \subset\{\sigma\}$. In the first case unilateral deviations are nonprofitable; some may be costly and others costless. In the second case all unilateral deviations are costly. Not surprisingly, strict equilibria, therefore, satisfy all the requirements for which the refinement literature has asked. In particular, every strict equilibrium is pure (a vertex of $\Delta$ ) and it is the unique best reply not only to itself but, by continuity of the payoff function, also to all strategy combinations in some neighborhood of itself. Formally, if $\sigma \in E(\Gamma)$ is strict, then there is some neighborhood $\mathscr{U}$ of $\sigma$ such that $\tilde{\beta}(\mathscr{U} \cap \Delta) \subset\{\sigma\}$. Hence, such an equilibrium is robust to all sufficiently small perturbations of the players' beliefs about each others' play.

The following lemma generalizes this observation, first, from the best-reply correspondence to all behavior correspondences, and, second, from individual strategy combinations to sets of strategy combinations. As a special case the result holds for all curb sets. (Note that the proof of Lemma 2 relies exclusively on the fact that behavior correspondences are closed mappings.)

LEMMA 2: If $X \in P$ is closed under some $\varphi \in \Phi$, then there exists a neighborhood $\mathscr{U}$ of $\Delta(X)$ such that $\varphi(\mathscr{U} \cap \Delta) \subset X$. 
Proof: Suppose $\varphi(\Delta(X)) \subset X$ and there is no neighborhood $\mathscr{U}$ of $\Delta(X)$ such that $\varphi(\mathscr{U} \cap \Delta) \subset X$. Let $Y$ be the complement of $X$ in $S$, and identify $X$ and $Y$ with the associated sets of vertices of $\Delta$. Then $X$ and $Y$ are disjoint closed subsets of $\Delta$. By hypothesis, $Y$ is nonempty and there exists a sequence $\left\{\sigma^{\tau}\right\}_{\tau=1}^{\infty}$ from $\Delta$ converging to some point $\sigma^{o} \in \Delta(X)$ such that $\varphi\left(\sigma^{\tau}\right)$ contains some point from $Y$, for all $\tau=1,2, \ldots$ Since $\varphi$ is u.h.c. and $Y$ is closed, this implies that also $\varphi\left(\sigma^{\circ}\right)$ contains some point from $Y$. But $Y$ is disjoint from $X$ and hence $\varphi\left(\sigma^{o}\right)$ is not a subset of $X$-a contradiction.

Q.E.D.

The next result establishes basic relationships between sets which are closed under some behavior correspondence and the set of Nash equilibria. Recall that the set $E(\Gamma) \subset \Delta$ of Nash equilibria of any normal-form game $\Gamma$ is the union of finitely many, disjoint, closed and connected sets, called connected components (Kohlberg and Mertens (1986, Proposition 1)). The following observation is trivially valid for any behavior correspondence $\varphi \in \Phi$ : Every connected component $C \subset E(\Gamma)$ is contained in the face $\Delta(X)$ spanned by some set $X \in P$ which is closed under $\varphi$ (just let $X=S$ ). Proposition 4(a) below establishes the partial converse that for any $X \in P$ which is closed under some behavior correspondence each connected component of Nash equilibria is either disjoint from or contained in the face spanned by $X$. Proposition 4(b) shows that every face spanned by a set $X \in P$ which is closed under some behavior correspondence contains a set of Nash equilibria which satisfies some of the strongest known set-wise refinement criteria, essentially (van Damme (1987, p. 266)), hyperstability, and strategic stability (Kohlberg and Mertens (1986, p. 1022 and p. 1027)). Since generic extensive-form games have only finitely many Nash equilibrium outcomes (Kreps and Wilson (1982, Theorem 2)), all strategy combinations in any connected set of Nash equilibria result in the same outcome in such games.

Proposition 4: (a) If $X \in P$ is closed under some behavior correspondence $\varphi \in \Phi$ and $C$ is a connected component of Nash equilibria, then either $C \subset \Delta(X)$ or $C \cap \Delta(X)=\varnothing$.

(b) If $X \in P$ is closed under some $\varphi \in \Phi$, then $\Delta(X)$ contains an essential connected component of Nash equilibria and, hence, a hyperstable set and $a$ strategically stable set of Nash equilibria.

Proof: (a) Suppose $X \in P$ is closed under $\varphi \in \Phi$, and let $C \subset E(\Gamma)$ be a connected component of Nash equilibria such that $C \cap \Delta(X) \neq \varnothing$. By Lemma 2 there exists a neighborhood $\mathscr{U}$ of $\Delta(X)$ such that $\varphi(\mathscr{U} \cap \Delta) \subset X$. Suppose $C$ is not a subset of $\Delta(X)$. Then there exists some $\sigma^{\circ} \in C \cap \mathscr{U}$ which does not belong to $\Delta(X)$. But $\beta\left(\sigma^{o}\right) \subset \varphi\left(\sigma^{o}\right) \subset X$, so $\sigma^{o} \notin \tilde{\beta}\left(\sigma^{o}\right)$, a contradiction to $\sigma^{o}$ being a Nash equilibrium.

(b) If $X \in P$ is closed under $\varphi \in \Phi$, then it is closed under $\beta \in \Phi$, by $\beta(\Delta(X)) \subset \varphi(\Delta(X)) \subset X$. Thus for all $\sigma \in \Delta(X)$ and all $i \in \mathscr{N}$

$$
s_{i}^{k} \notin X_{i} \Rightarrow U_{i}\left(\sigma_{-i}, s_{i}^{k}\right)<\max _{\hat{\sigma}_{i} \in \Delta_{i}} U_{i}\left(\sigma_{-i}, \hat{\sigma}_{i}\right) .
$$


By continuity (and the maximum theorem) there exists a neighborhood $\mathscr{O}$ of the game $\Gamma=(S, u)$ under consideration in the space of normal-form games $\Gamma^{\prime}=$ $(S, v)$ such that the above implication holds for all games in $\mathscr{O}$. Consequently, for all games $\Gamma^{\prime}=(S, v) \in \mathscr{O}$ one has $\beta_{v}(\Delta(X)) \subset X$, i.e., $X \in P$ is also closed under the best reply correspondence $\beta_{v}$ of the game $\Gamma^{\prime}$. The reduced game $\Gamma_{X}=(X, u)$, where players are restricted to the strategy spaces $X_{i}, \forall i \in \mathscr{N}$, has an essential component of Nash equilibria $C_{X} \subset E\left(\Gamma_{X}\right)$ (cf. Kohlberg and Mertens (1986, Proposition 1)). In other words: For every $\varepsilon>0$ there exists a neighborhood $\mathscr{O}_{X}^{\varepsilon}$ of $\Gamma_{X}=(X, u)$ such that for every $\Gamma_{X}^{\prime}=(X, v) \in \mathscr{O}_{X}^{\varepsilon}$ there exists some $\sigma^{\prime} \in E\left(\Gamma_{X}^{\prime}\right)$ within distance $\varepsilon$ from $C_{X} \subset \Delta(X)$ (in the Hausdorff metric). Then

$$
\mathscr{O}^{\varepsilon}=\left\{\Gamma^{\prime}=(S, v) \in \mathscr{O} \mid \Gamma_{X}^{\prime}=(X, v) \in \mathscr{O}_{X}^{\varepsilon}\right\}
$$

defines a neighborhood of $\Gamma=(S, u)$, and any $\Gamma^{\prime}=(S, v) \in \mathscr{O}^{\varepsilon}$ has some $\sigma^{\prime} \in$ $E\left(\Gamma_{X}^{\prime}\right)$ within distance $\varepsilon$ from $C_{X}$. But, since $\Gamma^{\prime} \in \mathscr{O}, \beta_{v}(\Delta(X)) \subset X$ and so $\sigma^{\prime} \in E\left(\Gamma^{\prime}\right)$. Moreover, $\beta_{u}(\Delta(X)) \subset X$ implies $C_{X} \subset E(\Gamma)$, so $C_{X}$ is an essential component for the game $\Gamma$. Every essential component contains a hyperstable set, and every hyperstable set contains a strategically stable set by standard arguments (cf. Kohlberg and Mertens (1986, p. 1022)).

Q.E.D.

An important role in the analysis below will be played by the "better-reply" correspondence $\gamma=\times_{i \in \mathscr{N}} \gamma_{i}: \Delta \rightarrow S$, defined by

$$
\gamma_{i}(\sigma)=\left\{s_{i} \in S_{i} \mid U_{i}\left(\sigma_{-i}, s_{i}\right) \geq U_{i}(\sigma)\right\}, \quad \forall i \in \mathscr{N} .
$$

Evidently $\gamma$ is u.h.c. and $\beta_{i}(\sigma) \subset \gamma_{i}(\sigma)$ for all players $i \in \mathscr{N}$ and strategy combinations $\sigma \in \Delta$, so $\gamma$ is a behavior correspondence.

In other words: $\gamma_{i}$ assigns to each strategy combination $\sigma \in \Delta$ those pure strategies $s_{i}$ which give at least the same payoff as $\sigma_{i}$. Such strategies $s_{i}$ are thus (weakly) better replies to $\sigma$ than $\sigma_{i}$ is. Moreover, $\gamma_{i}(\sigma)$ always contains some pure strategy from the support of $\sigma_{i}$. In particular, if $\sigma$ is a Nash equilibrium, then $\gamma_{i}(\sigma)$ contains the whole support of $\sigma_{i}$, and indeed one then has $\gamma(\sigma)=$ $\beta(\sigma)$. As a consequence, a singleton set $X=\{s\}$ is closed under $\gamma$ if and only if $s \in S$ is a strict equilibrium. More generally, this is true for all behavior correspondences the images of which are contained in the images of the "better-reply" correspondence:

CoRollary 1: If $\varphi \in \Phi$ is such that $\varphi(\sigma) \subset \gamma(\sigma), \forall \sigma \in \Delta$, then a singleton set $X=\{s\} \in P$ is closed under $\varphi$ if and only if $s \in S$ is a strict equilibrium.

PROOF: Lemma 1(c) covers the "only if" part. If $\sigma \in \Delta$ is a strict equilibrium, then $\sigma=s$ is pure and $\beta(s)=\gamma(s)=\{s\}$. Thus $\varphi(s)=\{s\}$ for all $\varphi \in \Phi$ which satisfy $\varphi(s) \subset \gamma(s), \forall s \in S$.

Q.E.D. 


\section{SET-WISE STABILITY}

We are now in a position to establish a characterization of asymptotic stability of faces of the polyhedron $\Delta$ of mixed strategy combinations, a characterization based exclusively on the data of the game under consideration. By applying this characterization no detailed information on the dynamics is needed in order to judge whether a given face is asymptotically stable or not; it is sufficient that the dynamics meets the conditions of a sign-preserving selection (SPS) dynamics. In particular, the class of asymptotically stable faces agrees for all such dynamics.

THEOREM 1: For any SPS dynamics and any set $X \in P$ : The face $\Delta(X)$ is asymptotically stable if and only if $X$ is closed under $\gamma$.

Proof: Suppose first $\gamma(\Delta(X)) \subset X$. Then there is some neighborhood $\mathscr{B}$ of $\Delta(X)$ such that $\gamma(\mathscr{B} \cap \Delta) \subset X$, by Lemma 2. There exists some $\varepsilon>0$ such that $\mathscr{B}$ contains the " $\varepsilon$-slice" $\mathscr{B}(\varepsilon)=\left\{\sigma \in \Delta \mid\right.$ inf $\left._{\bar{\sigma} \in \Delta(X)}\|\sigma-\bar{\sigma}\|<\varepsilon\right\}$. For any player $i \in \mathscr{N}$, let $Y_{i}$ be the complement to $X_{i}$ in $S_{i}$ and consider any SPS dynamics $\dot{\sigma}_{i}{ }^{k}=\sigma_{i}{ }^{k} f_{i}^{k}(\sigma), \forall k=1, \ldots, K_{i}, \forall i \in \mathscr{N}$. If $Y_{i}$ is empty, $\sigma_{i}\left(t, \sigma^{o}\right) \in \Delta_{i}\left(X_{i}\right)=\Delta_{i}$, $\forall \sigma^{o} \in \Delta, \forall t$. Otherwise, for every $s_{i}^{k} \in Y_{i}$ and $\sigma \in \mathscr{B}(\varepsilon)$ we have $U_{i}\left(\sigma_{-i}, s_{i}^{k}\right)<$ $U_{i}(\sigma)$, since $\gamma(\mathscr{B}(\varepsilon)) \subset X$. But this implies $\dot{\sigma}_{i}{ }^{k}<0$, for all $\sigma \in \mathscr{B}(\varepsilon)$ with $\sigma_{i}^{k}>0$. Hence $\sigma_{i}^{k}\left(t, \sigma^{o}\right) \rightarrow_{t \rightarrow \infty} 0, \forall \sigma^{o} \in \mathscr{B}(\varepsilon)$, implying $\sigma_{i}\left(t, \sigma^{o}\right) \rightarrow_{t \rightarrow \infty} \Delta_{i}\left(X_{i}\right)$. In order to establish the Lyapunov stability property of $\Delta(X)$ : For any neighborhood $\mathscr{B}^{\prime}$, let the neighborhood $\mathscr{B}^{\prime \prime}$ be such that $\mathscr{B}^{\prime \prime} \cap \Delta$ is an $\varepsilon^{\prime}$-slice $\mathscr{B}\left(\varepsilon^{\prime}\right) \subset$ $\mathscr{B}^{\prime} \cap \Delta$, and apply the above argument. This proves the "if" part.

Second, assume $X$ is not closed under $\gamma \in \Phi$. Then there is some pure strategy combination $\bar{s} \in X$, player $i \in \mathscr{N}$ and pure strategy $s_{i}^{k} \notin X_{i}$ such that $U_{i}\left(\bar{s}_{-i}, s_{i}^{k}\right) \geq U_{i}(\bar{s})$, since otherwise $U_{i}\left(s_{-i}, s_{i}^{\prime}\right)-U_{i}(s)<0, \forall s \in X, \forall i \in \mathscr{N}$, and $\forall s_{i}^{\prime} \in S_{i} \backslash X_{i}$, which would imply $U_{i}\left(\sigma_{-i}, s_{i}^{\prime}\right)-U_{i}(\sigma)<0, \forall \sigma \in \Delta(X), \forall i \in \mathscr{N}$, and $\forall s_{i}^{\prime} \in S_{i} \backslash X_{i}$, which is equivalent to $X$ being closed under $\gamma$. Let $s^{*}=$ $\left(\bar{s}_{-i}, s_{i}^{k}\right)$, and let $X^{*} \in P$ be defined by $X_{j}^{*}=\left\{\bar{s}_{j}\right\}, \forall j \neq i$, and $X_{i}^{*}=\left\{\bar{s}_{i}, s_{i}^{k}\right\}$, i.e., $\Delta\left(X^{*}\right)$ is the one-dimensional face (or edge) spanned by the two pure strategy combinations $\bar{s}$ and $s^{*}$. Moreover, $U_{i}\left(s^{*}\right)-U_{i}(\bar{s}) \geq 0$, and since $U_{i}$ is linear in $\sigma_{i}, U_{i}\left(\sigma_{-i}, s_{i}^{k}\right)-U_{i}(\sigma) \geq 0, \forall \sigma \in \Delta\left(X^{*}\right)$. Clearly $\Delta\left(X^{*}\right)$ is invariant under any SPS dynamics. Hence, for any initial state $\sigma^{o} \in \Delta\left(X^{*}\right)$ the solution path through $\sigma^{o}$ has $\dot{\sigma}_{i}^{k} \geq 0$, in any SPS dynamics, implying that $\sigma\left(t, \sigma^{o}\right)$ does not approach $\Delta(X)$ as $t \rightarrow \infty$. The two faces $\Delta\left(X^{*}\right)$ and $\Delta(X)$ having the point $\bar{\sigma}=\bar{s}$ in common implies that $\Delta(X)$ is not asymptotically stable.

Q.E.D.

REMARK 1: Suppose $X \in P$ is closed under $\gamma$ and consider an AMS dynamics with player-specific "shift-factors" $\omega_{i}, i \in \mathscr{N}$. For every $i \in \mathscr{N}$ and $s_{i}^{k} \in Y_{i}=S_{i} \backslash X_{i}$ let

$$
\delta_{i}^{k}=\inf _{\sigma \in \mathscr{B}(\varepsilon)} \omega_{i}(\sigma)\left[U_{i}(\sigma)-U_{i}\left(\sigma_{-i}, s_{i}^{k}\right)\right]
$$


where $\mathscr{B}(\varepsilon)$ is the " $\varepsilon$-slice" as in the proof of the Theorem. Since $\gamma(\Delta(X)) \subset X$, it follows for $\varepsilon>0$ sufficiently small that $\delta_{i}^{k}>0$. Therefore, $\dot{\sigma}_{i}^{k} \leq-\delta_{i}^{k} \sigma_{i}^{k}$, which implies that $\sigma_{i}^{k}(t) \leq \sigma_{i}^{k}(0) \exp \left\{-\delta_{i}^{k} t\right\}, \forall \sigma(0) \in \mathscr{B}(\varepsilon), \forall t \geq 0$. In this sense the weights assigned to pure strategies outside the set $X$ converge to zero exponentially in any AMS dynamics.

REMARK 2: Consider a symmetric two-player game. Let $\hat{\gamma}\left(\sigma_{1}\right)=\left\{s_{1} \in\right.$ $\left.S_{1} \mid U_{1}\left(s_{1}, \sigma_{1}\right) \geq U_{1}\left(\sigma_{1}, \sigma_{1}\right)\right\}$ and for any $X_{1} \subset S_{1}$ let $\hat{\gamma}\left(\Delta_{1}\left(X_{1}\right)\right)=\bigcup_{\sigma_{1} \in \Delta_{1}\left(X_{1}\right)} \hat{\gamma}\left(\sigma_{1}\right)$. The same arguments as in the first part of the proof of Theorem 1 can be used to show that if $X_{1} \subset S_{1}$ satisfies $\hat{\gamma}\left(\Delta_{1}\left(X_{1}\right)\right) \subset X_{1}$ then $\Delta_{1}\left(X_{1}\right)$ is asymptotically stable in any single-population sign-preserving selection dynamics.

One readily verifies, by the argument in the second part of the proof of Theorem 1 , that if $X \in P$ is not closed under $\gamma$, then $\Delta(X)$ cannot be asymptotically stable in any monotonic selection dynamics either (because $U_{i}\left(\bar{s}_{-i}, s_{i}^{k}\right) \geq U_{i}(\bar{s}), \bar{s}_{i}=s_{i}^{h}$, implies $f_{i}^{k}(\sigma) \geq f_{i}^{h}(\sigma), \forall \sigma \in \Delta\left(X^{*}\right)$, by $\sigma=\left(\bar{s}_{-i}, \sigma_{i}\right)$, $\forall \sigma \in \Delta\left(X^{*}\right)$, from monotonicity).

The other implication of Theorem 1 also generalizes to a wider class of dynamics. Suppose the dynamics is of the form $\dot{\sigma}_{i}^{k}=\xi_{i}^{k}(\sigma), \forall k=1, \ldots, K_{i}$, $\forall i \in \mathscr{N}$, for some Lipschitz continuous functions $\xi_{i}: \Delta \rightarrow \mathfrak{R}^{K_{i}}, \forall i \in \mathscr{N}$, which leave $\Delta$, but not necessarily all faces; positively invariant, and the dynamics is weakly sign-preserving (WSP) in the sense

$$
U_{i}\left(\sigma_{-i}, s_{i}^{k}\right)<U_{i}(\sigma) \Rightarrow \xi_{i}^{k}(\sigma)<0, \quad \forall s_{i}^{k} \in \operatorname{supp}\left(\sigma_{i}\right), \forall \sigma \in \Delta,
$$

for all $i \in \mathscr{N}$. Such WSP dynamics can be of interest when, due to mutations, say, the vector field $\xi$ points inwards at the boundary of $\Delta$. Applying the argument in the first part of the proof of Theorem 1, closure under $\gamma$ implies asymptotic stability in any WSP dynamics.

COROLlaRY 2: (a) If the face $\Delta(X)$ is asymptotically stable in some monotonic selection dynamics, then $X$ is closed under $\gamma$.

(b) If $X \in P$ is closed under $\gamma$, then $\Delta(X)$ is asymptotically stable in any WSP dynamics.

In view of Lemma 1(a) Theorem 1 implies that a set $X \in P$ is fixed under $\gamma$ if and only if the associated face $\Delta(X)$ is a minimal asymptotically stable face of the polyhedron $\Delta$, in any SPS dynamics. Furthermore, since a pure strategy combination $s \in S$, viewed as a singleton set, is fixed under the better-reply correspondence if and only if it is a strict equilibrium (Corollary 1), Theorem 1 also implies the following characterization of asymptotically stable pure strategy combinations:

COROLlARY 3: For any SPS dynamics: A pure strategy combination is asymptotically stable if and only if it is a strict equilibrium. 
Combining Theorem 1 with Proposition 4(b) yields that any face which is asymptotically stable in some SPS dynamics contains a closed and connected set of Nash equilibria which is essential, contains a hyperstable set, and hence a strategically stable set in the sense of Kohlberg and Mertens (1986):

COROllary 4: If a face $\Delta(X)$ is asymptotically stable in some SPS dynamics, then it contains an essential component of Nash equilibria, and thus also a hyperstable and a strategically stable set.

In the sense of set inclusion there is thus a link between asymptotic stability in evolutionary dynamics and strategic stability. The converse of Corollary 4 is trivial: Any strategically stable set, or essential component of Nash equilibria, is contained in some (minimal) face spanned by a set $X \in P$ which is closed under the better-reply correspondence.

As mentioned in the introduction, the claim that an asymptotically stable face of $\Delta$ contains a hyperstable subset also follows from Theorem 1 of Swinkels (1993). His result states that if a closed set $A \subset \Delta$ is (i) asymptotically stable in some myopic adjustment dynamics, a class which includes all SPS dynamics, and (ii) has a basin of attraction which contains a (relative) neighborhood of $A$ the closure of which is homeomorphic to $\Delta$, then $A$ contains a hyperstable subset. ${ }^{12}$ The topological condition (ii) is clearly met by all asymptotically stable faces of $\Delta$. Since a set is hyperstable (roughly) if it is minimal with respect to essentiality (cf. Kohlberg and Mertens (1986, p. 1022)), Corollary 4 also follows from Swinkels' result.

The hypothesis in Corollary 4 and in Swinkels' result, asymptotic stability of a given set, may, however, often be hard to verify. In such a case Theorem 1 is helpful, because it provides an operational necessary and sufficient condition for asymptotic stability, at least for a certain class of subsets of $\Delta$ and selection dynamics.

This raises the following issue: Which (closed) subsets of $\Delta$, other than its faces, are asymptotically stable in which class of selection dynamics? There is a trade-off here between the precision of evolutionary predictions, in the sense of "small" asymptotically stable sets, and the robustness of those predictions with respect to the dynamics. In many applications only broad qualitative features of the dynamics are known or assumed. So a fair amount of robustness of stability properties with respect to the details of the dynamics seems desirable. But such a robustness with respect to a subclass of selection dynamics may require relatively "large" sets.

In particular, it turns out that even a subclass of SPS dynamics, the AMS dynamics, is rich enough to disqualify all (closed) sets which belong to the

\footnotetext{
${ }^{12}$ Swinkels (1993) calls a regular dynamics a myopic adjustment dynamics if all Nash equilibria are rest points and $\sum_{k} \sigma_{i}^{k} f_{i}^{k}(\sigma) U_{i}\left(\sigma_{-i}, s_{i}^{k}\right) \geq 0$ holds, $\forall \sigma \in \Delta, \forall i \in \mathscr{N}$. It is not difficult to see that any SPS dynamics meets these requirements.
} 
relative interior of any of the faces of $\Delta \cdot{ }^{13}$ No such set can even be (Lyapunov) stable in all AMS dynamics, unless the relevant face consists entirely of rest points for all SPS and monotonic selection dynamics. Hence, one can disregard all relatively interior (closed) sets if one requires predictions to be robust across the class of AMS dynamics.

Proposition 5: If a closed set $A \subset \operatorname{int}(\Delta(X))$, for some $X \in P$, is (Lyapunov) stable in all AMS dynamics, then $\Delta(X) \subset F(\Gamma)$.

Proof: See Appendix.

An alternative robustness test is to require that the subsets under consideration be asymptotically stable in the benchmark case of the replicator dynamics. However, as pointed out in Section 3, no interior strategy combination is asymptotically stable in the replicator dynamics. The generalization of this claim to (relatively) interior closed sets is immediate. Therefore, to require asymptotic stability in the replicator dynamics again discards (relatively) interior closed sets.

PROPOSITION 6: No closed set in the (relative) interior of a face is asymptotically stable in the replicator dynamics.

Proof: See Appendix.

Clearly, when precise information on the dynamics is available, it is possible in some games to identify proper subsets of asymptotically stable faces which are themselves asymptotically stable or at least (Lyapunov) stable in that dynamics. This is the case, for instance, with the "matching pennies" game for which the solution paths to the replicator dynamics are closed orbits around the Nash equilibrium point. Although its unique (interior) Nash equilibrium constitutes a strategically stable singleton set, the only subset of $\Delta$ which is (Lyapunov) stable in all AMS dynamics is $\Delta$ itself. In fact, in this game the unique Nash equilibrium outcome is dynamically unstable in certain AMS dynamics which are arbitrarily close to the replicator dynamics (see the proof of Proposition 5 in the Appendix). Hence, in this sense, its (Lyapunov) stability in the replicator dynamics is not even locally robust in the space of AMS dynamics.

Another difference between strategic and evolutionary dynamic stability is that, though also some strategically stable sets may induce several outcomes (cf. Kohlberg and Mertens (1986, Figure 11)), for generic extensive-form games there exists at least one strategically stable set which induces a single outcome. However, as seen in the "matching pennies" game, a face which is asymptotically stable in all SPS dynamics need not correspond to a unique outcome, even if the face contains a strategically stable set which induces a single outcome.

\footnotetext{
${ }^{13}$ As with $\Delta$, the relative interior of a face $\Delta(X)$ is denoted by $\operatorname{int}(\Delta(X))$. A subset $A \subset \Delta(X)$ is called relatively interior, if it is a subset of $\operatorname{int}(\Delta(X))$.
} 
Hence, evolutionary processes may result in paths along which also the outcome persistently oscillates, even in nondegenerate games. For, while in a completely mixed Nash equilibrium each player is indifferent between all her pure strategies, she is required to randomize in a particular fashion in order to keep the other players "in equilibrium." In an evolutionary setting, however, there is no outside coordination mechanism, and individuals now and then change strategy in the light of little information. In particular, they need not know the payoffs associated with other player positions. Therefore, there is a priori nothing there to "bring them to order."

\section{EXAMPLES}

EXAMPLE 1: Figure 1 shows the (reduced) normal form of a game where player 1 first chooses whether to take an outside option $\left(s_{1}^{1}\right)$ or to move into a $2 \times 2$ subgame of the "battle-of-the-sexes" type that consists of the last two strategies for both players (van Damme (1989, Figure 1)). The game has two components of Nash equilibria, both of which contain subgame perfect equilibria: In the first component player 1 chooses her outside option and player 2 plays her first pure strategy with probability at least $1 / 3\left(\sigma_{1}^{1}=1,1 / 3 \leq \sigma_{2}^{1} \leq 1\right)$. The second component is the strict equilibrium, $\sigma_{1}^{2}=\sigma_{2}^{2}=1$. The first component does not contain a strategically stable set nor an equilibrium that satisfies forward induction in the sense of van Damme (1989, p. 485).

The game has three curb sets, $S, X=\left\{s_{1}^{2}\right\} \times\left\{s_{2}^{2}\right\}$, and $Y=\left\{s_{1}^{1}, s_{1}^{2}\right\} \times\left\{s_{2}^{1}, s_{2}^{2}\right\}$, but only $S$ and $X$ are closed under $\gamma$. By Theorem 1 the (singleton) set $X$ which induces the strategically stable outcome and satisfies forward induction in the sense of van Damme is the only asymptotically stable face (except for the full strategy set) in this game, for any SPS dynamics.

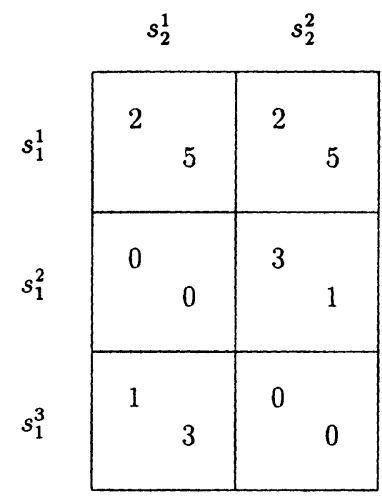

FiguRE 1. 


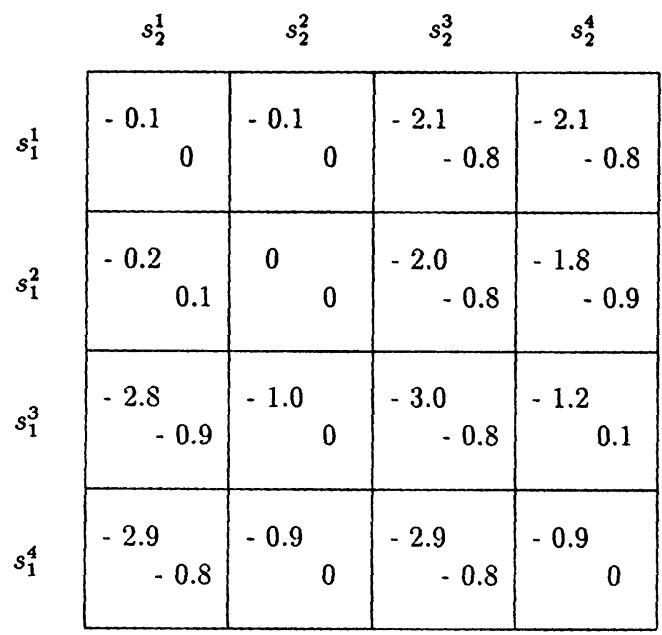

FIGURE 2.

EXAMPLE 2: Figure 2 shows the normal form of the "beer-quiche" signaling game due to David Kreps (Kohlberg and Mertens (1986, Figure 14, p. 1031); cf. also Cho and Kreps (1987, Figure 1)). In this game, player 1 observes a choice by nature assigning a type to her, "strong" with probability 0.9 and "weak" with probability 0.1 . Then she sends either a "strong" or a "weak" signal. Upon seeing the signal, player 2 decides whether to retreat or fight. Normal form strategies correspond to the following choices: Player 1 can either always send a "strong" signal $\left(s_{1}^{1}\right)$, or send a "strong" signal when she is strong and send a "weak" signal when she is weak $\left(s_{1}^{2}\right)$, or send a "weak" signal when she is strong and send a "strong" signal when she is weak $\left(s_{1}^{3}\right)$, or always send a weak signal $\left(s_{1}^{4}\right)$. Player 2 can either retreat upon seeing a "strong" signal and fight upon seeing a "weak" signal $\left(s_{2}^{1}\right)$, or always retreat $\left(s_{2}^{2}\right)$, or always fight $\left(s_{2}^{3}\right)$, or fight upon seeing a "strong" signal and retreat upon seeing a "weak" signal $\left(s_{2}^{4}\right)$. The game has two connected components of Nash equilibria. In the first, "good" component player 1 always sends a "strong" signal and player 2 retreats upon seeing a "strong" signal but fights with probability at least $1 / 2$ upon seeing a "weak" signal $\left(\sigma_{1}^{1}=1, \sigma_{2}^{1}+\sigma_{2}^{2}=1, \sigma_{2}^{1} \geq 1 / 2\right)$. In the second, "bad" component player 1 always sends a "weak" signal and player 2 retreats upon seeing a "weak" signal but fights with probability at least $1 / 2$ upon seeing a "strong" signal $\left(\sigma_{1}^{4}=1, \sigma_{2}^{2}+\sigma_{2}^{4}=1, \sigma_{2}^{4} \geq 1 / 2\right)$.

The only set $Y \in P$ which spans a face containing the second, "bad" component and which is closed under $\gamma$ is the whole strategy set $S$. On the other hand, the first, "good" component is contained in the face spanned be the set $X=\left\{s_{1}^{1}, s_{1}^{2}\right\} \times\left\{s_{2}^{1}, s_{2}^{2}\right\} \in P$, i.e., the set where player 1 either always sends a "strong" signal or sends a "strong" signal when she is strong and a "weak" signal when she is weak, and player 2 either retreats upon seeing a "strong" signal and fights upon seeing a "weak" signal or always retreats. From the 
payoffs in Figure 2 it is easy to verify that the set $X$ is closed under the better-reply correspondence and, in fact, is a fixed set both under $\gamma$ and $\beta$. So, in this example closure under $\gamma$ selects the strategy subset that contains the "intuitive" pure strategies.

EXAMPLE 3: The payoff bi-matrix in Figure 3 shows a two-player game with three strategies for each player. The game has three Nash equilibria, one of which (in the lower right corner) is strict. The other two are mixed, one being the equilibrium of the "matching-pennies" game that consists of the first two strategies for each player. Whether the set $X=\left\{s_{1}^{1}, s_{1}^{2}\right\} \times\left\{s_{2}^{1}, s_{2}^{2}\right\}$ is closed under $\gamma$ depends on the parameter $x$. If $x$ is negative, then the set $X$ is closed under $\gamma$, otherwise not. However, for all $x<2$ the $X$ is closed under $\beta$. If $x \geq 0$, then by Theorem 1 the face $\Delta(X)$ is not asymptotically stable in any SPS dynamics. Figure 4 illustrates some computer simulations of solution paths to the replicator dynamics starting near $\Delta(X)$ and converging to the strict equilibrium $s=\left(s_{1}^{3}, s_{2}^{3}\right)$. Here $x=1.9$, so $X$ is closed under $\beta$. In the diagram $p_{j}$, resp. $q_{j}$, denote the population shares using the $j$ th pure strategy, for $j=1,2,3$, for player roles 1 , resp. 2 . Note that the restriction of this game to mixed-strategy profiles in the face $\Delta(X)$ is, by itself, a constant-sum game with value $1-x / 2$. If $x$ is negative, then $X$ is closed under $\gamma$, and the constant-sum "subgame" has its own domain of attraction, just like a strict equilibrium. (In fact, the game then is a kind of generalized coordination game.)

EXAMPLE 4: As a final example, consider the three-player $3 \times 2 \times 2$ game of Figure 5, in which player 1 chooses tri-matrix, player 2 row, and player 3 column. For any fixed pure strategy of the first player, players 2 and 3 face a symmetric $2 \times 2$ game. When player 1 uses her first strategy $\left(s_{1}^{1}\right)$, the first strategies of players 2 and 3 ( $s_{2}^{1}$ and $s_{3}^{1}$, respectively) are strictly dominant. However, if players 2 and 3 would use those strategies, then player 1's best reply is to switch to her second strategy $\left(s_{1}^{2}\right)$. But when player 1 uses her second strategy, the

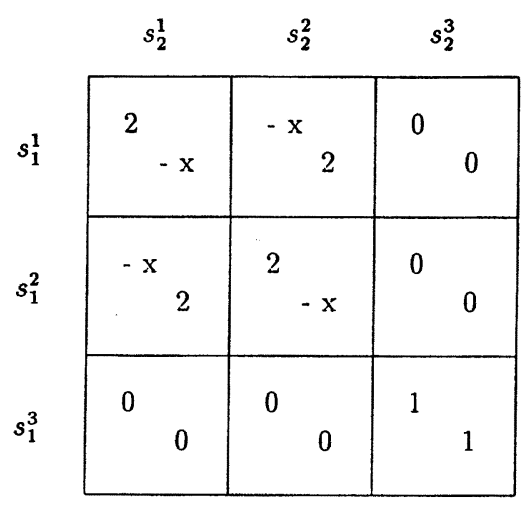

FIGURE 3. 


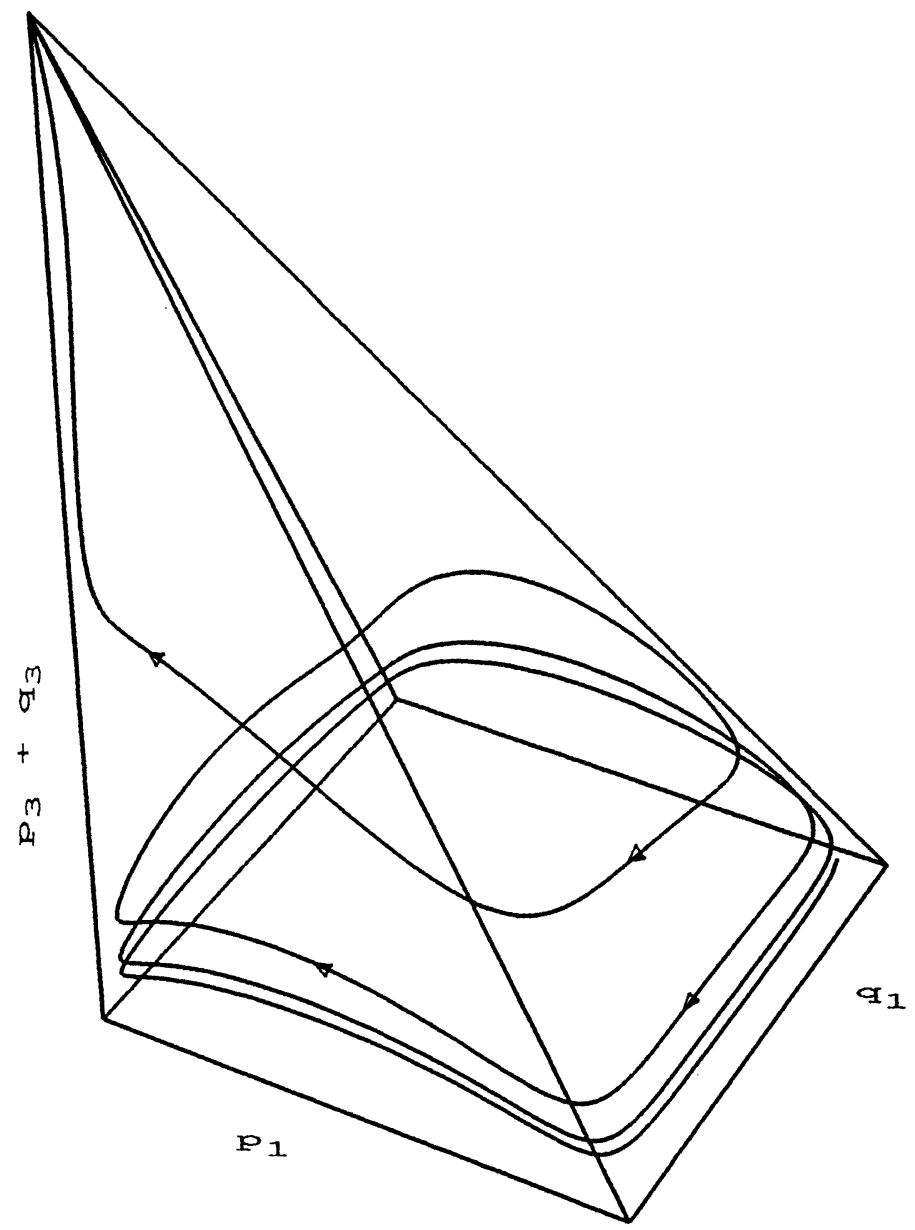

FigurE 4.

second strategies of players 2 and $3\left(s_{2}^{2}\right.$ and $\left.s_{3}^{2}\right)$ are strictly dominant, and if they would use these, player 1's best reply is her first strategy. When player 1 uses her third strategy $\left(s_{1}^{3}\right)$, finally, players 2 and 3 face a game of pure coordination.

It is not difficult to show that the product set $X=\left\{s_{1}^{1}, s_{1}^{2}\right\} \times S_{2} \times S_{3} \in P$ obtained by taking all players' first two strategies constitutes (the maximal) curb set, i.e., it is closed under $\beta$. But one can show that the excluded strategy, $s_{1}^{3}$, is not strictly dominated. Hence, it is a priori possible that the population share using strategy $s_{1}^{3}$ does not tend to zero along some interior solution paths. If this is the case even for (interior) trajectories starting arbitrarily close to the face spanned by $X$, then a set closed under $\beta$ need not even attract a neighborhood.

Indeed, computer simulations produce precisely such trajectories; see Figure 6 for solution orbits to the replicator dynamics. Since players 2 and 3 always 

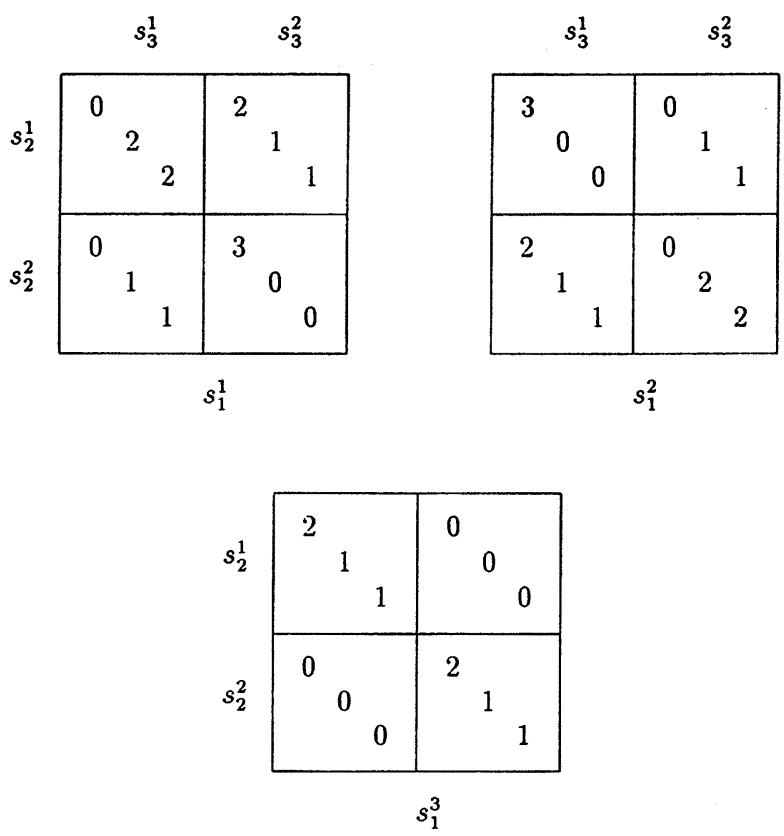

FiguRE 5 .

earn identical payoffs, the diagonal $\sigma_{2}=\sigma_{3}$ is invariant. The diagram shows a solution curve for which initially $\sigma_{1}(0)=(0.05,0.90,0.05)$, and $\sigma_{2}(0)=\sigma_{3}(0)=$ $(0.15,0.85)$, plotted in three-dimensional space with $\sigma_{1}^{1}$ on the "horizontal" axis, $\sigma_{1}^{2}$ on the "vertical" axis, and $\sigma_{2}^{1}=\sigma_{3}^{1}$ on the "depth" axis. The face spanned by $X$ is the sloping square. As one sees in this diagram, after a few initial rounds the solution curve swirls out towards a perpetual motion near the edges of the polyhedron, recurrently moving virtually as far away from the face spanned by $X$ as it is possible. ${ }^{14}$ So one peculiar feature of this example is that outcomes do not converge. The only trajectories that can be shown to converge to the face spanned by $X$ are those starting in the straight line segment $L=\left\{\sigma \in \Delta \mid \sigma_{2}^{1}=\right.$ $\left.\sigma_{3}^{1}=1 / 2, \sigma_{1}^{1}=\sigma_{1}^{2}\right\}$. This set is invariant and intersects the face spanned by $X$ at its mid-point, a Nash equilibrium which constitutes a (singleton) strategically stable set in the sense of Kohlberg and Mertens (1986).

This illustrates why evolutionary predictions may have to be less precise than, say, strategically stable sets of Nash equilibria are. All interior trajectories which do not start in $L$ will move outwards towards the edges of the polyhedron, but they will never come to a halt. Rather each such trajectory will visit each of the six vertices infinitely often. Since $L$ is lower-dimensional than the polyhedron shown in Figure 6, a prediction that concentrates on the Nash equilibrium will ignore the most likely results of evolution.

${ }^{14}$ The phenomenon illustrated by Figure 6 is known as a "heteroclinic cycle" in the theory of dynamical systems (cf. Sigmund (1992), Gaunersdorfer (1992)). 


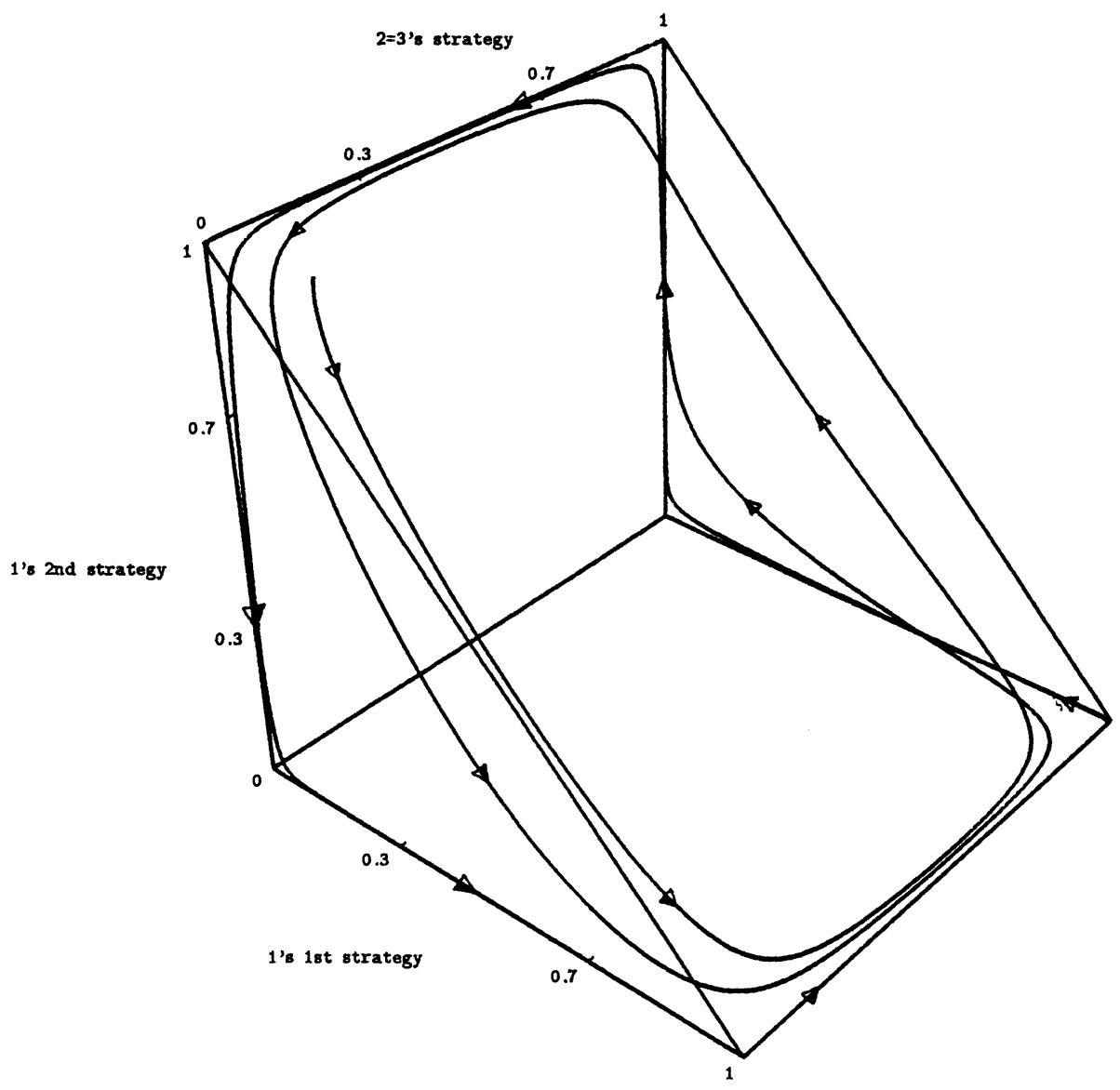

Figure 6.

7. DIRECTIONS FOR FURTHER RESEARCH

The results in the present paper suggest that set-wise criteria such as closure under a behavior correspondence may have stronger implications for dynamic evolutionary selection than the Nash equilibrium property has. This raises several further issues. Are similar methods capable of identifying faces which meet weaker stability criteria? Can the approach be generalized to a wider class of sets and/or dynamics (cf. Swinkels (1993))? For which classes of games do dynamic evolutionary approaches have strong cutting power? Can a similar approach be applied to the extensive form of the game (Nöldeke and Samuelson (1993))? Finally, deterministic selection dynamics in continuous time constitute only one possible formalization of evolutionary processes. It would be of great interest to explore the relation of closure under a behavior correspondence and, 
say, attractors in stochastic adaption processes for finite populations (see, e.g., Hurkens (1994)).

Institute for Advanced Studies, Department of Economics, Stumpergasse 56, A-1060 Vienna, Austria

and

Department of Economics, Stockholm School of Economics, P.O. Box 6501, 11383 Stockholm, Sweden.

Manuscript received June, 1993; final revision received November, 1994.

\section{APPENDIX}

Proof of Proposition 1: (a) Every Lyapunov stable state must be a rest point. Consider $\sigma \in F(\Gamma) \backslash E(\Gamma)$. There is some $i \in \mathscr{N}$ and $s_{i}^{k} \notin \operatorname{supp}\left(\sigma_{i}\right)$ such that $U_{i}\left(\sigma_{-i}, s_{i}^{k}\right)>U_{i}(\sigma)$, and hence $f_{i}^{k}(\sigma)>f_{i}^{h}(\sigma)=0, \forall s_{i}^{h} \in \operatorname{supp}\left(\sigma_{i}\right)$, by monotonicity and stationarity. By continuity of $f_{i}$ there is a neighborhood $\mathscr{O}$ of $\sigma$ such that $f_{i}^{k}\left(\sigma^{\prime}\right)>0, \forall \sigma^{\prime} \in \mathcal{O} \cap \Delta$. But this implies that $\sigma_{i}^{k}(t, \bar{\sigma})$ is strictly increasing in $t$, whenever $\bar{\sigma} \in \mathscr{O} \cap \Delta, \bar{\sigma}_{i}^{k}>0$, and $\sigma(t, \bar{\sigma}) \in \mathscr{O}$. Hence, either $\sigma_{i}^{k}(t, \bar{\sigma})$ increases forever, or $\sigma(t, \bar{\sigma}) \notin \mathscr{O}$ for some $t>0$. In both cases $\sigma$ is not Lyapunov stable, because $\sigma_{i}^{k}=0$.

(b) Suppose $\sigma^{o} \in \operatorname{int}(\Delta)$ and $\sigma\left(t, \sigma^{o}\right) \rightarrow_{t \rightarrow \infty} \sigma$. Then $\sigma \in F(\Gamma)$. If $\sigma \notin E(\Gamma)$, then, as in the proof of (a), there is $i \in \mathscr{N}, s_{i}^{k} \notin \operatorname{supp}\left(\sigma_{i}\right)$, and a neighborhood $\mathscr{O}$ of $\sigma$ such that $f_{i}^{k}\left(\sigma^{\prime}\right)>0$, $\forall \sigma^{\prime} \in \mathscr{O} \cap \Delta$. Convergence of $\sigma\left(t, \sigma^{o}\right)$ to $\sigma$ implies that there is some $T>0$ such that $\sigma\left(t, \sigma^{o}\right) \in \mathscr{O}$ $\cap \operatorname{int}(\Delta), \forall t \geq T$, because int $(\Delta)$ is invariant. But convergence to $\sigma$ and $\sigma_{i}^{k}=0$ imply that there is some $t>T$ such that $\dot{\sigma}_{i}^{k}<0$, which contradicts $f_{i}^{k}\left(\sigma^{\prime}\right)>0, \forall \sigma^{\prime} \in \mathscr{O} \cap \Delta$. Hence, $\sigma \in E(\Gamma)$. Q.E.D.

Proof of Proposition 2: Results in Sections 4 and 5 do not rely on Proposition 2 and can thus be used here.

(i) First assume that $\bar{\sigma} \in E(\Gamma)$ is asymptotically stable in the replicator equation. By Proposition 6 it must then be pure and by Corollary 3 it must be a strict equilibrium.

(ii) If $\bar{\sigma} \in E(\Gamma)$ is a strict equilibrium, then it is asymptotically stable by Corollary 3. Q.E.D.

Proof of Proposition 3: (a) Assume that there exists a neighborhood $\mathscr{\theta}$ of $\bar{\sigma}$ such that $\bar{\sigma} \in \tilde{\beta}\left(\sigma^{o}\right), \forall \sigma^{o} \in \mathscr{O} \cap \Delta$. Choose $\mathscr{O}^{\prime} \subset \mathcal{O}$ to be a neighborhood of $\bar{\sigma}$ such that $\mathscr{O}^{\prime} \cap \Delta$ is convex and $\bar{\sigma}_{i}^{k}>0 \Rightarrow \sigma_{i}^{k}>0, \forall \sigma \in \mathscr{O}^{\prime} \cap \Delta, \forall k=1, \ldots, K_{i}, \forall i \in \mathscr{N}$. Define the function $V_{\bar{\sigma}}: \mathscr{O}^{\prime} \cap \Delta \rightarrow \Re_{+}$ by

$$
V_{\bar{\sigma}}(\sigma)=-\sum_{i \in \mathscr{N}} \sum_{k=1}^{K_{i}} \bar{\sigma}_{i}^{k} \ln \left(\sigma_{i}^{k}\right) \geq 0,
$$

which is continuously differentiable on $\mathscr{O}^{\prime}$. By Jensen's inequality, $\sigma \neq \bar{\sigma}$ implies

$$
V_{\bar{\sigma}}(\sigma)-V_{\bar{\sigma}}(\bar{\sigma})=-\sum_{i \in \mathscr{N}} \sum_{k=1}^{K_{i}} \bar{\sigma}_{i}^{k} \ln \left({\sigma_{i}}^{k} / \bar{\sigma}_{i}^{k}\right)>-\sum_{i \in \mathscr{N}} \ln \left(\sum_{k=1}^{K_{i}} \bar{\sigma}_{i}^{k} \frac{\sigma_{i}^{k}}{\bar{\sigma}_{i}^{k}}\right)=0,
$$

so $\sigma=\bar{\sigma}$ is the unique minimum of $V_{\bar{\sigma}}$.

Taking the time derivative of $V_{\bar{\sigma}}$ yields

$$
\dot{V}_{\bar{\sigma}}(\sigma)=\frac{d}{d t} V_{\bar{\sigma}}(\sigma)=-\sum_{i \in \mathscr{N}} \sum_{k=1}^{K_{i}} \bar{\sigma}_{i}^{k} f_{i}^{k}(\sigma) \leq 0,
$$


because $\bar{\sigma}_{i}^{k}>0 \Rightarrow s_{i}^{k} \in \beta(\sigma), \forall \sigma \in \mathscr{O}^{\prime} \cap \Delta$, hence $U_{i}\left(\sigma_{-i}, s_{i}^{k}\right) \geq U_{i}(\sigma)$, which by the definition of SPS dynamics implies $f_{i}^{k}(\sigma) \geq 0, \forall s_{i}^{k} \in \operatorname{supp}(\bar{\sigma}), \forall i \in \mathscr{N}$. Hence, $V_{\bar{\sigma}}$ is a local Lyapunov function, implying that $\bar{\sigma}$ is a stable rest point for any SPS dynamics.

(b) If the SPS dynamics is specialized to an AMS dynamics, then from robustness it follows now that $\dot{V}_{\bar{\sigma}}(\sigma)=0$ implies $U_{i}\left(\sigma_{-i}, \bar{\sigma}_{i}\right)=U_{i}(\sigma), \forall i \in \mathscr{N}$, such that $\sigma \in \tilde{\beta}(\sigma)$, and hence $\sigma \in E(\Gamma)$. Thus $V_{\bar{\sigma}}(\sigma)<0$ for all $\sigma \in \mathscr{O}^{\prime} \cap \Delta$ which satisfy $\sigma \notin E(\Gamma)$. Therefore, $\sigma\left(t, \sigma^{o}\right) \rightarrow_{t \rightarrow \infty} E(\Gamma) \cap \mathcal{O}^{\prime}, \forall \sigma^{o} \in$ $\mathscr{O}^{\prime} \cap \Delta$, as required.

Q.E.D.

Proof of Proposition 5: Since each face of $\Delta$ is invariant in every regular selection dynamics, and the restriction of such a dynamics to any face is a regular selection dynamics with respect to the associated reduced game, we may assume without loss of generality that $X=S$.

Consider an AMS dynamics $\dot{\sigma}_{i}^{k}=f_{i}^{k}(\sigma) \sigma_{i}^{k}$ and call the "shift-factors" $\omega_{i}$ from the representation of AMS dynamics given in Section 2.2 payoff increasing if there exists a continuously differentiable function $g_{i}: \Re \rightarrow \Re_{++}$with positive derivative, $g_{i}^{\prime}>0$, such that $\omega_{i}(\sigma)=g_{i}\left(U_{i}(\sigma)\right)$, $\forall \sigma \in \Delta$.

Claim 1: If a closed set $A \subset \operatorname{int}(\Delta)$ with nonempty interior is positively invariant in an AMS dynamics with payoff increasing "shift-factors" $\omega_{i}, \forall i \in \mathscr{N}$, then $\Delta=F(\Gamma)$ :

Define the vector field $\zeta$ on $\operatorname{int}(\Delta)$ by

$$
\zeta_{i}^{k}(\sigma)=\frac{\sigma_{i}^{k} f_{i}^{k}(\sigma)}{P(\sigma)}, \text { where } P(\sigma)=\prod_{j \in \mathscr{N}} \prod_{h=1}^{K_{j}} \sigma_{j}^{h},
$$

for all $k=1, \ldots, K_{i}, \forall i \in \mathscr{N}$. Because $P(\sigma)>0$ on int $(\Delta)$, multiplying the vector field associated with the AMS dynamics by $P(\sigma)^{-1}$ does not alter the solution curves of the AMS dynamics in $\operatorname{int}(\Delta)$. Thus the differential equation $\dot{\sigma}=\zeta(\sigma)$ has the same rest points and the same stability properties on $\operatorname{int}(\Delta)$ as the original AMS dynamics. Henceforth let $\hat{\sigma}\left(t, \sigma^{o}\right)$ denote a solution to $\dot{\sigma}=\zeta(\sigma)$ through $\sigma^{o}=\hat{\sigma}\left(0, \sigma^{o}\right) \in \operatorname{int}(\Delta)$.

Define the directional derivative, at some point $\sigma_{i} \in \operatorname{int}\left(\Delta_{i}\right)$, of a differentiable function $g: \operatorname{int}\left(\Delta_{i}\right)$ $\rightarrow \mathfrak{A}$ in the direction towards a vertex $s_{i}^{k}$ of $\Delta_{i}$ by

$$
\partial g\left(\sigma_{i}, s_{i}^{k}\right)=\frac{\partial g\left(\sigma_{i}\right)}{\partial \sigma_{i}^{k}}-\sigma_{i} \cdot \operatorname{grad}\left(g\left(\sigma_{i}\right)\right)
$$

If $\omega_{i}$ is differentiable, $\forall i \in \mathscr{N}$, by the explicit representation of AMS dynamics one obtains

$$
\begin{aligned}
\partial \zeta_{i}^{k}\left(\sigma, s_{i}^{k}\right)= & P(\sigma)^{-1}\left[\omega_{i}(\sigma)\left(K_{i}-2\right)\left[U_{i}\left(\sigma_{-i}, s_{i}^{k}\right)-U_{i}(\sigma)\right] \sigma_{i}^{k}\right. \\
& \left.+\sigma_{i}^{k}\left[U_{i}\left(\sigma_{-i}, s_{i}^{k}\right)-U_{i}(\sigma)\right] \partial \omega_{i}\left(\sigma, s_{i}^{k}\right)\right] .
\end{aligned}
$$

The divergence of a vector field is the trace of its Jacobian, so

$$
\operatorname{div} \zeta(\sigma)=P(\sigma)^{-1} \sum_{i \in \mathscr{N}} \sum_{k=1}^{K_{i}} \sigma_{i}^{k}\left[U_{i}\left(\sigma_{-i}, s_{i}^{k}\right)-U_{i}(\sigma)\right] \partial \omega_{i}\left(\sigma, s_{i}^{k}\right),
$$

at any $\sigma \in \operatorname{int}(\Delta)$. If the player-specific "shift-factors" $\omega_{i}$ are payoff increasing, then

$$
\operatorname{div} \zeta(\sigma)=P(\sigma)^{-1} \sum_{i \in \mathscr{N}} g_{i}^{\prime}\left(U_{i}(\sigma)\right) \sum_{k=1}^{K_{i}}\left[U_{i}\left(\sigma_{-i}, s_{i}^{k}\right)-U_{i}(\sigma)\right]^{2} \sigma_{i}^{k} \geq 0,
$$

for all $\sigma \in \operatorname{int}(\Delta)$. Clearly, $\operatorname{div} \zeta(\sigma)>0, \forall \sigma \in \operatorname{int}(\Delta) \backslash F(\Gamma)$, and $\operatorname{div} \zeta(\sigma)=0, \forall \sigma \in \operatorname{int}(\Delta) \cap F(\Gamma)$.

Suppose the closed set $A \subset \operatorname{int}(\Delta)$ with $\operatorname{int}(A) \neq \varnothing$ is positively invariant in the AMS dynamics with payoff increasing "shift-factors." Assign to $A$ the volume $V_{o}=\int_{A} d \sigma>0$, because $\operatorname{int}(A) \neq \varnothing$, define $A(t)=\left\{\sigma \in \Delta \mid \sigma=\hat{\sigma}\left(t, \sigma^{o}\right), \sigma^{o} \in A\right\}$ and $V(t)=\int_{A(t)} d \sigma$. If $A$ is positively invariant, then $A(t) \subset A \subset \operatorname{int}(\Delta), \forall t \geq 0$. By Liouville's theorem (see, e.g., Hofbauer and Sigmund (1988, pp. 170, 
281)) the volume $V(t)$ of $A(t)$ satisfies

$$
\dot{V}(t)=\int_{A(t)} \operatorname{div} \zeta(\sigma) d \sigma \geq 0, \quad \forall t \geq 0 .
$$

Therefore, $V(t) \geq V_{o}>0, \forall t \geq 0$. But, because $A$ is positively invariant, also $V_{o} \geq V(t), \forall t \geq 0$, such that $\dot{V}(t)=0, \forall t \geq 0$. Hence $V_{o}=\int_{A \cap F(\Gamma)} d \sigma$, since $\operatorname{div} \zeta(\sigma)>0, \forall \sigma \in A \backslash F(\Gamma)$. Because $V_{o}>0$, it follows that $A \cap F(\Gamma)$ must contain an open set (with strictly positive volume). However, $F(\Gamma)$ is the set of zeros of the replicator equation (the AMS dynamics with $\omega_{i}(\sigma)=1, \forall \sigma \in \Delta, \forall i \in \mathscr{N}$ ) and, therefore, the set of solutions of a polynomial equation. A system of polynomial equations which vanishes on an open nonempty set vanishes everywhere and, consequently, $F(\Gamma)=\Delta$. This establishes Claim 1.

ClAIM 2: If a closed set $A \subset \operatorname{int}(\Delta)$ is (Lyapunov) stable in all AMS dynamics, then $\Delta=F(\Gamma)$ :

If the closed set $A \subset \operatorname{int}(\Delta)$ is (Lyapunov) stable in all AMS dynamics, then it must be stable in an AMS dynamics with payoff increasing "shift-factors." By definition, for every neighborhood $\mathscr{B}$ ' of $A$ there exists a neighborhood $\mathscr{B} "$ of $A$ such that

$$
\mathscr{B}(t)=\left\{\sigma \in \Delta \mid \sigma=\sigma\left(t, \sigma^{o}\right), \sigma^{o} \in \mathscr{B}^{\prime \prime} \cap \Delta\right\} \subset \mathscr{B}^{\prime}, \quad \forall t \geq 0,
$$

where $\sigma\left(t, \sigma^{\circ}\right)$ denotes the solution to an AMS dynamics with payoff increasing "shift-factors." Choose $\mathscr{B}^{\prime}$ such that closure $\left(\mathscr{B}^{\prime} \cap \Delta\right) \subset \operatorname{int}(\Delta)$ and define $\mathscr{B}_{\infty}=\bigcup_{t \geq 0} \mathscr{B}(t)$. Then $B \equiv \operatorname{closure}\left(\mathscr{B}_{\infty}\right)$ $\subset \operatorname{int}(\Delta)$ and $\mathscr{B}_{\infty}$ is a (relative) neighborhood of $A$, because for every $t \geq 0$ the set $\mathscr{B}(t)$ is (relatively) open as the image of the (relatively) open set $\mathscr{B}^{\prime \prime} \cap \Delta$ under the continuous one-to-one mapping $\bar{\sigma} \mapsto \sigma(t, \bar{\sigma})$, and $\mathscr{B}_{\infty}$ is (relatively) open as the union of (relatively) open sets $\mathscr{B}(t)$, and $A \subset \mathscr{B}_{\infty}$ by construction. But $\mathscr{B}_{\infty}$ is positively invariant as the union of (forward) orbits. Since with $\mathscr{B}_{\infty}$ also its closure $B$ is positively invariant, $B$ is a closed set with nonempty (relative) interior which is contained in $\operatorname{int}(\Delta)$ and positively invariant in the AMS dynamics with payoff increasing "shift-factors." Consequently, Claim 1 implies $\Delta=F(\Gamma)$.

Q.E.D.

Proof of Proposition 6: Again we may assume without loss of generality $\Delta(X)=\Delta$. Consider the replicator dynamics, i.e., the AMS dynamics with $\omega_{i}(\sigma)=1, \forall \sigma \in \Delta, \forall i \in \mathscr{N}$. Define the vector field $\zeta$ on $\operatorname{int}(\Delta)$ as in the proof of Proposition 5 by multiplying the vector field associated with the replicator dynamics by $P(\sigma)^{-1}$. Then $\partial \omega_{i}\left(\sigma, s_{i}^{k}\right)=0, \forall \sigma \in \Delta, \forall s_{i}^{k} \in S_{i}, \forall i \in \mathscr{N}, \operatorname{implies} \operatorname{div} \zeta(\sigma)=0$, $\forall \sigma \in \operatorname{int}(\Delta)$. If a compact set $A \subset \operatorname{int}(\Delta)$ is asymptotically stable in the replicator dynamics, then it is so for $\dot{\sigma}=\zeta(\sigma)$. Denote by $\hat{\sigma}\left(t, \sigma^{o}\right)$ a solution to $\dot{\sigma}=\zeta(\sigma)$ with $\hat{\sigma}\left(0, \sigma^{o}\right)=\sigma^{o} \in \operatorname{int}(\Delta)$. Let $\mathscr{B} \subset \operatorname{int}(\Delta)$ be a relative neighborhood of $A$ with compact closure $\overline{\mathscr{B}} \subset \Delta$ such that $\sigma^{o} \in \overline{\mathscr{B}} \Rightarrow$ $\hat{\sigma}\left(t, \sigma^{o}\right) \rightarrow_{t \rightarrow \infty} A$. Define $\mathscr{B}(t)=\left\{\sigma \in \Delta \mid \sigma=\hat{\sigma}\left(t, \sigma^{o}\right), \sigma^{o} \in \mathscr{B}\right\}$. Assign to $\mathscr{B}$ volume $V(0)=\int_{\mathscr{B}} d \sigma$ $>0$ and to $A$ volume $V_{o}=\int_{A} d \sigma$.

Suppose for some $\varepsilon>0$ there exists no finite time after which $\mathscr{B}(t)$ is within (Hausdorff) distance $\varepsilon$ from $A$ and remains there. Then there exists an increasing sequence $\left\{t_{k}\right\}_{k=1}^{\infty}$ with $t_{k} \rightarrow_{k \rightarrow \infty}+\infty$ and initial states $\left\{\sigma^{k}\right\}_{k=1}^{\infty}, \sigma^{k} \in \overline{\mathscr{B}}, \forall k$, such that the (Hausdorff) distance between $\sigma\left(t_{k}, \sigma^{k}\right)$ and $A$ is at least $\varepsilon$, for all $k$. Since $\overline{\mathscr{B}}$ is compact, the sequence $\left\{\sigma^{k}\right\}_{k=1}^{\infty}$ contains a convergent subsequence, so we can assume that it converges to $\sigma^{*} \in \overline{\mathscr{B}}$. By the hypothesis of asymptotic stability the distance from $\sigma\left(t_{k}, \sigma^{*}\right)$ to $A$ converges to zero. Thus there is some $k^{\prime}$ such that $\sigma\left(t_{k}, \sigma^{*}\right)$ is within distance $\varepsilon / 2$ from $A$, for all $k \geq k^{\prime}$. Since the the solution mapping is continuous in initial conditions and $A$ is Lyapunov stable, $\sigma\left(t_{k}, \sigma^{k}\right)$ is within distance $\varepsilon$ from $A$, for some $k \geq k^{\prime}-$ a contradiction.

Consequently, for every $\varepsilon>0$ there is some finite time after which the whole set $\mathscr{B}(t)$ is within (Hausdorff) distance $\varepsilon$ from the set $A$ and remains there. Thus $V(t)=\int_{\mathscr{B}(t)} d \sigma \rightarrow_{t \rightarrow \infty} V_{o}<V(0)$ implying that for some $t$ one must have $\dot{V}(t)<0$. But the latter contradicts

$$
\dot{V}(t)=\int_{\mathscr{B}(t)} \operatorname{div} \zeta(\sigma) d \sigma=0, \quad \forall t \geq 0,
$$

by Liouville's theorem. 


\section{REFERENCES}

Amann, E., AND J. HofBauer (1985): "Permanence in Lotka-Volterra and Replicator Equations," in Lotka-Volterra Approach to Cooperation and Competition in Dynamical Systems, ed. by W. Ebeling and M. Peschel. Berlin: Akademie-Verlag.

Aumann, R., AND A. Brandenburger (1992): "Epistemic Conditions for Nash Equilibrium," unpublished, Hebrew University and Harvard Business School.

Basu, K., AND J. W. Weibull (1991): "Strategy Subsets Closed Under Rational Behavior," Economics Letters, 36, 141-146.

BERGE, C. (1963): Topological Spaces. New York: MacMillan.

Binmore, K., AND L. Samuelson (1994): “Drift,” European Economic Review, 38, 859-867.

BJÖRNERSTEDT, J., AND J. W. WeIBUll (1993): "Nash Equilibrium and Evolution by Imitation," unpublished, Stockholm University.

BomZE, I. M. (1986): “Non-Cooperative Two-Person Games in Biology: A Classification,” International Journal of Game Theory, 15, 31-57.

CHO, I.-K., AND D. M. KREPS (1987): "Signaling Games and Stable Equilibria," Quarterly Journal of Economics, 102, 179-221.

Friedman, D. (1991): “Evolutionary Games in Economics," Econometrica, 59, 637-666.

Friedman, M. (1953): "The Methodology of Positive Economics," in Essays in Positive Economics, M. Friedman. Chicago: University of Chicago Press.

$\rightarrow$ Gaunersdorfer, A. (1992): “Time Averages for Heteroclinic Attractors," Siam Journal for Applied Mathematics, 52, 1476-1489.

HARSANYI, J. (1973): "Games with Randomly Disturbed Payoffs: A New Rationale for Mixed-Strategy Equilibrium Points," International Journal of Game Theory, 2, 1-23.

Hirsch, M. W., ANd S. Smale (1974): Differential Equations, Dynamical Systems, and Linear Algebra. New York: Academic Press.

Hofbauer, J., AND K. Sigmund (1988): The Theory of Evolution and Dynamical Systems. Cambridge: Cambridge University Press.

Hurkens, S. (1994): "Learning by Forgetful Players: From Primitive Formations to Persistent Retracts," CentER Working Paper No. 9437, Tilburg.

KohlBerG, E., AND J.-F. MerTens (1986): “On the Strategic Stability of Equilibria,” Econometrica, 54, $1003-1037$.

KREPS, D. M., AND R. Wilson (1982): “Sequential Equilibrium,” Econometrica, 50, 863-894.

MAYNARd SMith, J. (1982): Evolution and the Theory of Games. Cambridge: Cambridge University Press.

Maynard Smith, J., And G. Price (1973): “The Logic of Animal Conflict,” Nature, 246, 15-18.

NACHBAR, J. H. (1990): “'Evolutionary' Selection Dynamics in Games: Convergence and Limit Properties,” International Journal of Game Theory, 19, 59-90.

NASH, J. (1950): "Non-Cooperative Games," Dissertation at Princeton University.

Nöldeke, G., AND L. SAMUelson (1993): “An Evolutionary Analysis of Backward and Forward Induction," Games and Economic Behavior, 5, 425-454.

OKadA, A. (1983): "Robustness of Equilibrium Points in Strategic Games," unpublished, Tokyo Center for Game Theory.

Ritzberger, K., AND K. Vogelsberger (1990): “The Nash Field,” IAS Research Report No. 263, Vienna.

SAmuelson, L., AND J. Zhang (1992): "Evolutionary Stability in Asymmetric Games,” Journal of Economic Theory, 57, 363-391.

Sigmund, K. (1992): “Time Averages for Unpredictable Orbits of Deterministic Systems," Annals of Operations Research, 37, 217-228.

SwinKELS, J. (1992a): "Evolutionary Stability with Equilibrium Entrants," Journal of Economic Theory, 57, 306-332. 
(1992b): "Evolution and Strategic Stability: From Maynard Smith to Kohlberg and Mertens," Journal of Economic Theory, 57, 333-342.

- (1993): "Adjustment Dynamics and Rational Play in Games," Games and Economic Behavior, 5, 455-484.

TAN, T. C.-C. AND S. R. D. C. Werlang (1988): "The Bayesian Foundations of Solution Concepts of Games," Journal of Economic Theory, 45, 370-391.

TAYLOR, P. D. (1979): "Evolutionary Stable Strategies with Two Types of Players," Journal of Applied Probability, 16, 76-83.

TAYLOR, P. D., AND L. B. Jonker (1978): "Evolutionary Stable Strategies and Game Dynamics," Mathematical Biosciences, 40, 145-156.

Tномаs, B. (1985): “On Evolutionarily Stable Sets,” Journal of Mathematical Biology, 22, 105-115. VAN DAMmE, E. (1987): Stability and Perfection of Nash Equilibria. Berlin: Springer-Verlag. $476-496$.

ZeEman, E. C. (1980): Population Dynamics from Game Theory, Lecture Notes in Mathematics 819. Berlin: Springer-Verlag. 